\title{
Belphégor
}

\section{Genèse et structure du discours critique de Jean- Christophe Menu}

\section{Benjamin Caraco}

\section{(2) OpenEdition}

\section{Journals}

\section{Electronic version}

URL: http://journals.openedition.org/belphegor/811

DOI: 10.4000/belphegor.811

ISSN: 1499-7185

\section{Publisher}

LPCM

\section{Electronic reference}

Benjamin Caraco, « Genèse et structure du discours critique de Jean-Christophe Menu », Belphégor [Online], 14 | 2016, Online since 31 October 2016, connection on 02 May 2019. URL : http:// journals.openedition.org/belphegor/811 ; DOI : 10.4000/belphegor.811

This text was automatically generated on 2 May 2019.

\section{(c) (†) $\odot$}

Belphégor est mis à disposition selon les termes de la Licence Creative Commons Attribution - Pas d'Utilisation Commerciale - Pas de Modification 4.0 International. 


\title{
Genèse et structure du discours critique de Jean-Christophe Menu
}

\author{
Benjamin Caraco
}

1 Avec le recul, le renouveau de la bande dessinée francophone initié dans les années 1990 n'apparait plus uniquement comme le seul fait des éditeurs alternatifs. Pour Erwin Dejasse, les acteurs les plus visibles de celui-ci ont contribué à éclipser la diversité du paysage des auteurs et éditeurs impliqués. Cette renaissance suivait pourtant des logiques originales, notamment en termes géographiques. ${ }^{1}$ La bande dessinée en dissidence du groupe de recherche ACME, dont est tirée la contribution de Dejasse, s'efforce ainsi de réinscrire les évolutions du champ de la bande dessinée de ces vingt dernières années dans une perspective plus globale.

2 Cette entreprise salutaire ne doit pas faire perdre de vue que certains auteurs et éditeurs ont compté plus que d'autres à la fois quantitativement (succès économique) et qualitativement (réception critique). Les acteurs les plus visibles vont jusqu'à incarner le renouveau et contribuent - volontairement ou non - à l'émergence d'un nouveau standard (le roman graphique) et d'une nouvelle conception de la bande dessinée, qui en finissent par recouvrir la diversité des œuvres produites à la même époque. Cette entreprise de simplification de l'histoire du champ s'explique en partie par le passage par le filtre médiatique qui a tendance à amplifier les succès, notamment économiques. Toutefois, la compréhension du phénomène ne peut pas se résumer uniquement à cette dimension.

3 Comment rendre compte par exemple du rôle joué par L'Association dans ce renouveau ? Par quels moyens cette maison d'édition en est-elle arrivée à incarner la «nouvelle bande dessinée " alors que la majorité des ouvrages cités en référence lorsque l'on parle de celle-ci ne figurent pas à son catalogue $?^{2}$ De même, son ancienneté, bien qu'elle ait contribué à sa notoriété, n'a sûrement pas été aussi décisive que cela. En effet, de nombreuses structures analogues naissent à la même époque. Ne pouvons-nous pas émettre l'hypothèse selon laquelle l'un des aspects décisifs de sa contribution à ce renouveau repose sur la production d'un discours alternatif sur la bande dessinée, en 
accompagnement des œuvres éditées ? Cet élément contribuerait à expliquer la position singulière de L'Association au sein du champ de la bande dessinée.

4 L'Association, maison d'édition fondée par un collectif d'auteurs, a été à l'origine d'un important discours sur le médium, porté principalement par Jean-Christophe Menu, dont le rôle fut également moteur dans l'animation éditoriale. D'autres auteurs de L'Association se sont exprimés au sujet de la bande dessinée, mais le plus souvent lors d'entretiens dont ils ne sont pas à l'initiative et jamais avec une même constance et détermination. L'écrasante majorité du discours produit par L'Association est l'œuvre de Menu. Dans ses bandes dessinées et écrits, qu'ils soient de l'ordre du paratexte (de l'accompagnement aux œuvres comme les catalogues, les éditoriaux de Lapin, le Rab de Lapin puis Les Nouvelles de l'Hydre) ${ }^{3}$ ou du texte (l'essai Plates-bandes, ${ }^{4}$ ses articles parus dans L'Éprouvette et sa thèse de doctorat $L a$ Bande dessinée et son double ${ }^{5}$ ), Menu s'est efforcé de construire un discours cohérent sur la bande dessinée et ses potentialités. Pour Menu, il était justifié de lui accorder le statut d'art à part entière et de lui reconnaitre une place plus élevée dans la hiérarchie culturelle. Pour cela, il était nécessaire de dépasser et de dénoncer ses fortunes éditoriales dominantes, que Menu accusait d'occulter la richesse potentielle de la bande dessinée.

5 Pour comprendre les choix qui ont guidé l'aventure éditoriale de L'Association et la production de certaines œuvres, il semble nécessaire de s'arrêter sur l'arsenal argumentatif mis en œuvre par Menu, avant et pendant la période où il a présidé à la destinée de la maison d'édition, en tant que primus inter pares puis comme seul maître à bord après 2006, année de l'explosion du comité éditorial d'origine. L'une des caractéristiques de ce discours est sa production consciente. ${ }^{6}$ Loin d'être purement théorique, le discours de Jean-Christophe Menu souligne l'importance de la pratique et de son regard d'auteur et d'éditeur à l'encontre d'autres réflexions contemporaines, comme celles de Thierry Groensteen ou Benoît Peeters, qui sont avant tout le produit de théoriciens plus que de créateurs, notamment pour le premier. Cet élément déterminant se traduit (parfois) par un mode d'expression particulier - l'utilisation de la bande dessinée pour parler de la bande dessinée - et par une réflexion qui rend indissociable critique, théorie et pratique. En conséquence, celle-ci ne se focalise pas seulement sur la dimension esthétique de la bande dessinée. Elle envisage aussi les aspects politicoéconomiques via l'alimentation d'une polémique au sein du champ.

6 L'analyse du discours d'un auteur-éditeur de premier plan apporte un éclairage nouveau sur les acteurs œuvrant pour une meilleure reconnaissance de la bande dessinée comme art et comme forme culturelle légitime. Les travaux consacrés aux discours sur la bande dessinée ont en effet davantage privilégié les écrits des non-praticiens - en toute logique, puisqu'ils étaient les principaux producteurs de ce type de discours et ce jusqu'aux années 1970-1980. Pour Harry Morgan, les « auteurs sont les grands absents dans la production du discours sur la bande dessinée (alors qu'en littérature écrite, les écrivains ont évidemment été les premiers théoriciens de leur pratique). $»^{7}$

7 La contribution la plus éclairante et la plus globale sur «Les discours sur la bande dessinée par ses créateurs » est à ce jour le fait d'Erwin Dejasse, même s'il convient de signaler les articles de Tanguy Habrand ${ }^{8}$ et Björn-Olav Dozo ${ }^{9}$ sur des aspects particuliers. Dans son article, Dejasse s'interroge sur un paradoxe fondateur de la bande dessinée : « Si a priori la théorisation sur un art à peine éclos aurait de quoi surprendre, on peut s'étonner encore davantage qu'un fossé temporel de plus d'un siècle sépare l'Essai de physiognomonie des textes ultérieurs. ${ }^{10} \mathrm{Il}$ avance deux hypothèses afin d'expliquer 
cette absence : «le déficit de légitimité culturelle » et « l'absence d'autonomie du champ artistique de la bande dessinée ", sans expliciter si cette absence d'autonomie se fait au profit du champ économique ou d'un champ artistique plus large. Sa réflexion ne prend pas non plus en compte les caractéristiques sociales des auteurs, notamment leur niveau d'éducation. Dejasse brosse un tableau des freins à l'émergence d'un tel discours (le long compagnonnage entre bande dessinée et jeunesse), des occasions manquées (le groupe des auteurs de Charlie) comme des tentatives restées confidentielles (STP). Ce n'est que dans les années 1990 avec la montée en puissance d'une bande dessinée autobiographique que le discours sur le médium, produit par des auteurs, trouve un premier support. Cette nouvelle génération d'auteurs « commente abondamment sa propre pratique » et au sein de ce groupe, Dejasse souligne le rôle singulier et déterminant de Jean-Christophe Menu.

A partir des écrits de Jean-Christophe Menu, nous nous interrogerons sur l'évolution de ses propos et sur les conditions de sa réception. Qu'est-ce qui fait que son discours s'impose par rapport aux précédentes tentatives ? Son discours est-il en accord avec les œuvres éditées par L'Association alors qu'il prône la pratique comme démonstration de la valeur artistique de la bande dessinée ? La spécificité du discours de Menu repose-t-elle sur cet appel à la pratique ou avant tout sur le recours à la polémique et à la lutte ? En quoi son positionnement en tant qu'éditeur, critique et auteur contribue-t-il à diffuser son discours? Une telle démarche soulignerait une conscience du champ beaucoup plus aiguë que chez ses prédécesseurs. Son discours ne reposerait-il pas simplement sur une combinaison inédite de pratique et de polémique? Notons ici que seuls ses écrits qui appartiennent à la catégorie "texte» précédemment évoquée retiendrons notre attention ce qui revient à ne pas traiter dans le cadre de cette analyse tout ce qui relève de l'autobiographie en bande dessinée, et dans une moindre mesure, du paratexte (catalogue, éditoriaux en tout genre).

9 Ce discours, en dépit de sa grande cohérence sur plus de deux décennies, ne peut être appréhendé en un bloc et connaît des évolutions. Du milieu des années 1980 au tout début des années 1990 et de L'Association, il se veut programmatique et d'une certaine façon didactique même s'il est d'ores et déjà vindicatif. Du milieu des années 1990 au milieu des années 2000 - l'essai Plates-bandes constituant un jalon - le discours s'éclipse au profit de la pratique, c'est-à-dire à des œuvres, mais continue à exister en creux au fil du paratexte produit par L'Association. La dernière moitié des années 2000 constitue le sommet du discours de Jean-Christophe Menu sur la bande dessinée. Son discours devient à la fois plus polémique et systématique. S'il fait alors preuve d'une grande activité individuelle, il combine celle-ci avec l'organisation de la production d'un discours collectif sur la bande dessinée via la revue et la collection "Eprouvette». Au terme de ce développement historique, il conviendra de s'interroger sur la singularité éventuelle du discours de JeanChristophe Menu sur la bande dessinée.

\section{Avant et aux débuts de L'Association (1985-1990) : des écrits programmatiques et didactiques}

10 Faut-il voir dans l'intérêt pour le discours critique sur la bande dessinée de JeanChristophe Menu la trace de son passage par l'UFR (Unité de Formation et de Recherche) d'arts plastiques de l'université Paris-1 ? La « vocation » de la bande dessinée semble en effet bien ancrée chez Menu et cela dès son plus jeune âge. Il n'en allait pas de même pour la poursuite d'études supérieures comme il a pu en témoigner. Cette rencontre fortuite 
entre un désir et un environnement relâché où l'accent était mis sur la réunion entre théorie, critique et pratique, aura une importance capitale sur Jean-Christophe Menu. Outre que son passage à l'université lui permet de quitter "l'ornière un peu exclusivement $\mathrm{BD}$ » dans laquelle il pouvait être engagé à l'époque, son parcours n'aura alors de cesse de concilier réflexion et pratique. ${ }^{11}$

\section{Ecrits de jeunesse (1985-1988)}

11 Les écrits qui parsèment Le Lynx, prolongement de l'importante production enfantine et adolescente de fanzines de Jean-Christophe Menu, constituent les premières prises de position du jeune auteur. Les éditoriaux du Lynx font preuve d'une confiance étonnante, qui se traduit par la mise en avant assurée des contributions réunies, de la fierté et un discours assez vindicatif sur le renouvellement de la bande dessinée $\left(n^{\circ} 7\right)$; l'histoire du jeune collectif est mise en récit, l'éditorial du $n^{\circ} 6$ parle de "mythe " et d' " anthologie " concernant les œuvres présentes; enfin, l'humour caractérise ces propos se prenant pourtant au sérieux. En sus de ces ouvertures, les chroniques de «Jack Trotski» proposent un ensemble de brèves égrainées sur le mode de la conservation, presque anecdotique (et que l'on retrouvera dans le style de certains Rabs), qui ne sont pas sans rappeler la "Gazette» de Frémion dans le magazine Fluide Glacial. "Copinages " et contre-publicités sur les titres à éviter cohabitent avec la provocation comme lorsque les rédacteurs s'amusent à remettre des prix «Meder » pour les plus mauvaises bandes dessinées de l'année. Ces chroniques forment un espace contrôlé de circulation des publications sur la bande dessinée et esquissent une communauté de bandes dessinées correspondant aux goûts des contributeurs du fanzine. L'autocritique est aussi présente puisque lorsque certains travaux de Menu sont mentionnés il est précisé « qu['il] ne s'est pas foulé » $\left(n^{\circ} 7\right.$, p. 55). Le fanzine comporte des entretiens, dans le $n^{\circ} 6$ avec Chaland et Edika. La première interview, menée par Menu et Stanislas, est plus révérencieuse que la seconde, Menu étant cette fois accompagné de Thiriet. Le choix de ces deux auteurs assez différents illustre l'hétérogénéité des contributeurs de la jeune revue. Dans le $n^{\circ} 7$, c'est Masse qui est l'objet de leurs attentions : ce dernier est perçu par les rédacteurs comme un auteur de bande dessinée aux inclinaisons littéraires et aux préoccupations avant tout artistiques. Outre cette interview, le numéro est complété par un questionnaire envoyé à des cadres de l'édition sur les rapports qu'entretiennent création et publication. Les réponses, plus ou moins élaborées, émanent de Fillipini, Thévenet, Pasamonik et Diament. Les questions font montre d'un intérêt certain pour la structuration du champ de la bande dessinée et la politique éditoriale des maisons en présence.

Globof (ou Glob-off), publié durant ces mêmes années à l'occasion du festival d'Angoulême, est complémentaire du Lynx, dont il porte le nom en sous-titre. La matière éditoriale de l'éphémère quotidien ressemble aux chroniques de Jack Trotski : il annonce événements et concerts du Off, publications d'auteurs et éditeurs amis comme Dupuy et Berberian ou la collection X de Futuropolis. Produit collectivement et rapidement par l'ossature de ce que deviendra L'Association, le journal affiche sa spontanéité et ne se départit jamais d'un ton très ironique comme avec le « Manifeste de quinze » (1988) à la syntaxe écorchée qui se moque de l'ignorance du fan de « BD » moyen. Les Globof sont en majorité constitué de dessins des collaborateurs du journal (principalement des caricatures ou des strips) accompagnés de textes humoristiques qui dénoncent en creux les travers du festival et de sa population: course aux dédicaces et aux originaux et plus généralement 
" collectionnite ", mise en avant d'une bande dessinée racoleuse, instrumentalisation à des fins purement touristiques de l'événement par la municipalité d'Angoulême... Le journal brise le consensus (perçu) lors de la grande fête de la bande dessinée en s'en prenant notamment à quelques têtes de turcs. Henri Filippini (Glénat/Circus) ou Michel Greg sont particulièrement voués aux gémonies. Presque plus qu'avec Le Lynx, l'humour se veut ici délirant, absurde et potache. Le détournement fait son apparition et permet de dénoncer par le biais du ridicule un système dans lequel ces auteurs ne se retrouvent pas.

13 À la fin des années 1980, Menu fait ses premiers pas comme critique. Il participe aux Cahiers de la bande dessinée alors sous la houlette de Thierry Groensteen. Menu entre par la petite porte puisqu'il commet initialement des comptes rendus dans la rubrique «Le Crible » à partir du nº 76 (1987). Toutefois, contrairement à certaines critiques qui se contentaient de résumer l'histoire, Menu propose une lecture graphique des albums qu'il recense. Il n'hésite pas à commenter la qualité de l'édition, l'objet en lui-même, le tout dans un style littéraire. Dans sa critique d'In Vitro de Caro (Ed. Hoebeke), Menu offre un modèle de critique qui privilégie la forme (graphique) plutôt que le fond (le récit) et qui vise à distinguer l'originalité dans la production de bande dessinée. Dans le nº 80 (1988), il livre une analyse poussée du Journal d'Henriette en axant sa démonstration sur la contribution de Dupuy et Berberian au renouvellement du genre. De même, il prend la peine de situer historiquement et graphiquement le style de l'auteur espagnol Miguel Angel Prado $\left(n^{\circ} 80,1988\right)$. Dans le $n^{\circ} 77$ à propos d'un album qui n'a pas vraiment retenu son attention, Menu se livre à un exercice de détestation en parodiant le phrasé d'un adolescent à la recherche de divertissement («Et j'l'ai balancé par la fenêtre, c'te daube, j'te jure ! Ça m'apprendra à acheter d'la BD, alors là !»). Outre ses comptes rendus, Menu illustre le « Bloc-Notes » de Thierry Groensteen à partir du n 79 (1988) et jusqu'au départ de ce dernier. Dans ces pages, Menu s'adonne alors au genre de la vignette et propose un florilège grinçant annonciateur, en moins lisse, du Contre la bande dessinée de Gerner. Beaucoup de ses dessins se focalisent sur la réception de la bande dessinée considérée comme un art plus que limité. Enfin, dans le tout dernier numéro des Cahiers dirigé par Groensteen ( $\left.n^{\circ} 83\right)$, Menu participe de façon conséquente à l'imposant dossier consacré au scénariste Yann avec plusieurs études sur son œuvre et une participation à l'interview de l'auteur. Une telle contribution appelle plusieurs commentaires. L'attention portée à Yann semble s'expliquer par la tentative de renouvellement du magazine Spirou par le scénariste. Au sein d'une institution classique, Yann (en compagnie de Conrad) apporte une note subversive à un journal alors en perte de vitesse selon Menu. Ce dernier propose une histoire cyclique des fortunes de Spirou. Menu livre une analyse bibliographique méticuleuse et documentée des interventions de Yann dans les pages du magazine, notamment via les « Hauts de page ». La contribution de Yann est envisagée de manière duale comme à la fois destructive (« les hauts-de-page qui cherchaient à jeter la lumière sur toutes les tromperies du journal», p. 83) et constructive avec la série des "Innommables ». Ce renouvellement subversif ne peut être toutefois mené à terme, au grand regret de Menu. Dans son "Yann scénariste paradoxal ", Menu souligne les principales caractéristiques de l'auteur : «la provocation», «le sens du tragique », «la perspective historique » et le " goût de la référence. » Difficile de ne pas lire en creux une partie des préoccupations du jeune auteur («Tout, chez Yann, semble relever du paradoxal, du conflictuel. », p. 90). Menu achève son dernier grand article sur Yann en soulignant: "Cette importance accordée au Passé, dont j'ai relevé les diverses manifestations, place toute l'œuvre de Yann sous le signe de la nostalgie. Une nostalgie ambiguë, bien sûr, car la recherche d'un passé idéalisé va de pair avec un regard ironique 
et distanciateur.» (p. 109). Un des grands thèmes qui traverse la réflexion de Menu se trouve ici introduit.

14 Ces années d'études, de fanzinat et de critique sont également celle d'un « apprentissage » à l'ombre d'Etienne Robial, éditeur de Futuropolis, comme le rappelle Florence Cestac. ${ }^{12}$ Menu fréquente la librairie du couple et Robial ne tarde pas à lui faire confiance en lui proposant l'animation d'une revue. Le parcours du fondateur de Futuropolis a une influence déterminante sur Menu. Moins loquace que ne le sera son élève, qui republiera tout de même dans le premier numéro L'Éprouvette l'une de ses prises de position («La Bande Dessinée se meurt, merci la critique!», paru initialement en 1981), Robial incarne une forme de discours par ses pratiques éditoriales reprises à L'Association. Dans un entretien donné aux Cahiers de la bande dessinée en 1985 ( n $^{\circ}$ 62), Robial expose sa politique éditoriale. Celle-ci se caractérise par la place donnée aux auteurs, notamment via la collection «30/40» qui met en avant le nom de l'artiste ou encore la recherche de nouveaux talents avec la collection « $\mathrm{X} »$; une politique patrimoniale incarnée par la collection «Copyright »; et plus généralement une grande attention portée au livre comme objet, qui s'explique en partie par la double casquette d'éditeur et de graphiste de Robial. Enfin, chose assez rare pour l'époque, Futuropolis publie quelques ouvrages théoriques sur la bande dessinée comme celui de Bruno Lecigne.

Très tôt dans la carrière de Jean-Christophe Menu, trois activités se trouvent liées : celles d'auteur, d'éditeur et de critique. Son discours n'est pas encore constitué et navigue entre le bravache des débuts et une sensibilité critique aiguë, tous deux accompagnés d'une touche d'humour. Ces premiers pas dans ces domaines préfigurent un discours qui commencera à se cristalliser à la charnière des années 1980 et 1990.

\section{Un programme (1988-1990)}

\section{Un premier manifeste : la revue Labo}

L'unique numéro de la revue Labo, lancé avec l'aide du même Robial, date de 1990 et comprend deux réflexions critiques de Menu sur la bande dessinée. La première est un éditorial de deux pages sous la forme d'une bande dessinée. Il insiste de façon imagée sur le besoin de renouveau du champ, fondé sur un retour aux sources. Menu, par l'intermédiaire d'un narrateur à la tête noire barrée d'un X blanc, ${ }^{13}$ lance implicitement un appel à la nouvelle génération d'auteurs, représentée par un jeune dinosaure : il faut éviter de reproduire le massacre des années 1980 symbolisé par de grands dinosaures s'entre-déchirant, métaphore du marasme éditorial de l'époque, et par une foule de personnages résumant les maîtres-mots des intrigues de la bande dessinée de l'époque (sexe et violence). Ce sont là deux planches hautement symboliques mais qui doivent moins retenir notre attention que l'article qui clôt presque la revue : "L'Art de tous les paradoxes $"{ }^{14}$ Cet article condense et synthétise une grande partie du discours qui sera ensuite développé par Menu à la fois en parole et en acte; il joue le rôle de manifeste d'une conception de la bande dessinée ; enfin, il ne néglige pas la dimension réflexive et autocritique de l'engagement militant de Menu.

17 L'état éditorial de la bande dessinée dans les années 1980 joue d'ores et déjà le rôle de matrice de la révolte et du militantisme qui caractérisera durablement le discours de Menu. Ce dernier voit dans cette décennie comme le gâchis des acquis des années 
1960-1970. Parmi ces acquis, l'on retrouvait une forme de reconnaissance des possibilités de la bande dessinée par un public cultivé. Afin de capter ce succès, une logique économique et gestionnaire de l'édition s'est alors installée avec la complicité de certains auteurs et sous le regard dépité d'autres. Au lieu du passage à l'âge adulte promis, la bande dessinée serait rentrée dans une adolescence prolongée. Ce discours de la dénonciation s'accompagne d'une nostalgie d'un âge d'or de la bande dessinée mais aussi d'une ouverture à l'internationale (Raw d'Art Spiegelman). L'enjeu majeur consisterait à détacher la vraie bande dessinée de sa forme économique dégradée. Concrètement, cela se traduit par la fin de l'œcuménisme ambiant et par la nécessité de distinguer et donc de définir ce que devrait être la bande dessinée. ${ }^{15}$ Pour Menu, la tâche aurait été compliquée par l'association entre bande dessinée et contre-culture qui aurait favorisé sa marginalisation, sa spécialisation (avec des circuits de librairies propres notamment) et in fine sa relégation dans un "ghetto" sous-culturel. Les acteurs du monde de la bande dessinée doivent donc s'engager dans une démarche réflexive afin de prendre conscience que la bande dessinée ne se réduit pas à la standardisation en vigueur. Les principaux éléments de son discours sont posés.

Toutefois, cet article est d'autant plus remarquable que son auteur s'épanche sur le rapport ambivalent qu'il entretient avec la critique de la bande dessinée, entre le rejet d'une critique vécue comme parasite et l'exaltation d'un discours théorique perçu comme indispensable au développement de la bande dessinée en tant qu'art. Sur un plan individuel, Menu voit ainsi la critique comme une tâche chronophage susceptible d'éloigner de la création, sans compter qu'elle n'incite guère à l'optimisme. Sa critique se nourrit parfois d'une « aigreur » et lui donne régulièrement la sensation de « rabâcher ». Bien que la simple publication d'un tel texte fournisse déjà une partie de la réponse, ${ }^{16}$ Menu s'estime tiraillé entre ces deux possibilités. ${ }^{17}$ Si Menu adoptera sur la longue durée la seconde attitude, elle n'ira pas sans générer des tensions avec son activité d'auteur, celle d'éditeur pouvant être considérée plus directement comme une traduction en actes de ce « militantisme ».

\section{Un programme personnel : le mémoire de maîtrise}

Les positions affichées par Menu dans cet article de 1990 synthétisent une réflexion plus profonde menée à l'occasion de la rédaction d'un mémoire de maîtrise sous la direction de Bernard Teyssèdre en 1988. Sous le titre de Journal d'une existence de Bandes Dessinées, ${ }^{18}$ Menu livre un travail universitaire manuscrit mêlant écriture, cases et planches de bande dessinée dans une veine rappelant les écrits de Töpffer consacrés à la littérature en estampes. Cette forme s'explique aussi bien par des contingences temporelles que par un parti-pris d'authenticité. Le caractère "manuscrit», "linéaire» et improvisé (sans recours au brouillon) du texte s'inspire du Manifeste du surréalisme d'André Breton. ${ }^{19}$ Pour Menu, il s'agit de livrer, en bon surréaliste, une « exploration, basée sur l'existence d'une pratique et d'un rapport intime au médium concerné, [qui] est avant tout une profession de foi (ou de manque de foi). $»^{20}$ Dans son mémoire, Menu interroge à la fois la crise éditoriale du secteur et sa crise personnelle concernant le bien-fondé de sa pratique d'auteur, qu'il n'hésite pas à questionner. Ses nombreuses interventions graphiques le représentent continuellement en train de s'autocritiquer et symbolisent le doute qui entoure sa pratique de la bande dessinée. Dès son mémoire de maîtrise, Menu considère la bande dessinée comme une écriture et envisage un temps de réaliser entièrement celui-ci sous la forme d'une bande dessinée avant d'en abandonner l'idée. 
dans la plupart de ses écrits, la réflexion est éminemment personnelle : Menu fait le point sur son parcours jusqu'ici et s'interroge sur la compatibilité entre ses casquettes d'auteur et de critique en se demandant s'il est possible d'être à la fois juge et partie. Dès cette époque, il est conscient d'un paradoxe qui lui sera parfois reproché : très polémique dans ses prises de position, dénonciateur du conformisme ambiant, il n'en produit pas moins des œuvres de facture classique. D'un côté, il prêche pour l'expérimentation, et de l'autre, il dessine de «l'aventure». Comment expliquer ce paradoxe? Une distinction entre académisme et classicisme ne permet-elle pas d'en rendre raison? Menu la justifie par son inconscient nostalgique.

Dans ce mémoire, qui préfigure la structure de sa thèse, Menu alimente ses réflexions par sa pratique personnelle. Il opère une distinction dans ses œuvres entre celles qui s'inscrivent dans l'héritage, comme celles associées à son personnage « Lapot », et celles qui détruisent celui-ci, « Meder » en premier lieu. Menu ne refuse pas en bloc le passé de la bande dessinée, au contraire, il critique avant tout les auteurs « académiques » qui ne font que reproduire des recettes éculées sans apporter rien de neuf au médium. Il oppose ainsi la bande dessinée "classique » à une bande dessinée " de recherche ». Par exemple, son ami Stanislas s'inscrit dans le "mythe » de la bande dessinée contrairement à un Mattt Konture ancré dans "l'anti-mythe». Menu se situe à mi-chemin entre ces deux auteurs. En effet, il part de sa nostalgie pour la bande dessinée de son enfance (aussi bien lue que produite par l'enfant qu'il était ${ }^{21}$ ) pour en faire un espace de liberté créatrice. Menu estime d'abord créer pour lui sans intégrer la contrainte du regard extérieur, c'està-dire le «malaise » suscité par la probable publication : il y a un «» hic » entre plaisir de créer et désir de communiquer $\gg .{ }^{22}$ Dans cette optique, la contrainte externe (notamment économique) est niée au profit d'une contrainte interne librement choisie et qui s'inspire des principes du surréalisme. À cet effet, Menu compare la bande dessinée à une «sorte d'écriture automatique $» .^{23}$ Plus largement, ce mémoire se veut être un appel à une expérimentation inscrite dans un héritage, ce qui annonce son parcours ultérieur. Les personnages de «Lapot » et «Meder » font de Menu un auteur " polymorphe » pouvant s'essayer à des styles ambivalents. Menu énonce ainsi ses ambitions: réaliser des exercices référentiels, mathématiques et collectifs.

La dimension éditoriale n'est pas absente puisqu'il fait déjà part de sa volonté de publier un comix underground, de s'éditer aussi bien que de jouer ce rôle pour d'autres afin de donner un nouveau souffle à la bande dessinée. Lorsqu'il écrit ces lignes, l'entreprise est déjà lancée, que cela soit avec le Lynx à tifs, puis le Lynx et l'AANAL. ${ }^{24}$ Menu appelle de ses vœux une bande dessinée qui ne cherche pas à répondre à la demande de ses lecteurs, bande dessinée incarnée à ses yeux par le Glénat de Filippini. Dans les dernières pages de son mémoire, il s'estime convaincu de l'existence d'une "alternative » à la bande dessinée des années 1980 et souhaite contribuer à cette lutte et à faire émerger un « mouvement » participant à ce renouveau. Avec ce mémoire de maîtrise, Menu se place clairement dans une perspective programmatique. Il formalise finalement un effort déjà enclenché aussi bien individuellement - comme auteur - que collectivement - comme éditeur de fanzines. Enfin, il se lance dans une autocritique qui caractérise une partie de son œuvre écrite, qu'il s'agisse de sa thèse ou de ses interventions dans la Munographie qui lui sera consacrée. 


\section{Une approche didactique : Le cours de bande dessinée}

Le cours de bande dessinée réalisé par Menu en 1990 pour le Centre National d'Enseignement à Distance (CNED) ${ }^{25}$ s'apparente aux tentatives d'Eisner ${ }^{26}$ et de $\mathrm{McCloud}^{27}$. Pendant « cinq ou six ans ", Menu sera professeur de bande dessinée par correspondance et, outre ce cours, rédigera et corrigera des planches données en exercice. La didactique a bien sûr sa place dans son ouvrage mais celui-ci se démarque de la neutralité attendue d'un professeur. Ce Cours de bande dessinée prend en effet la forme d'une anthologie et d'un éloge des possibilités de création du médium. Le cours d'un peu moins d'une centaine de planches s'organise autour du plan suivant: "repères historiques", " approche ontologique ", "bases techniques » et une "conclusion » qui se refuse à en être une.

Dans la première partie, Menu brosse une rapide histoire de la bande dessinée qui débute avec Yellow Kid et enchaîne sur Little Nemo. Töpffer est cependant évoqué comme un précurseur. Pour Menu, la bande dessinée semble implicitement commencer avec le phylactère et ses codes sont maîtrisés dès ses débuts avec des auteurs comme Windsor McCay ou George Herriman. Il ne faut pas attendre longtemps avant que le récit historique de Menu ne se double d'une approche critique revendiquée : la bande dessinée d'aventure est présentée comme un genre empruntant une grande partie de ses repères au cinéma, ce qui la rendrait facilement transposable et donc non spécifique. On retrouve ici l'idée de «story-board » de Barthélémy Schwartz et la dénonciation du " genre » en bande dessinée qui éloignerait cette dernière de son essence. Le cours d'histoire se transforme donc en cours de critique, les deux étant finalement indissociables. Menu, autocritique, se moque ainsi de la nostalgie pour la bande dessinée franco-belge des années 1960 même s'il reconnaît, via son alter ego, le narrateur à la tête noire barrée d'un $\mathrm{X}$, y succomber également. Dans la chronologie de Menu, la fin des années 1960 constitue un tournant avec le magazine Pilote mais surtout avec l'arrivée d'Hara-Kiri/Charlie, puis dans les années 1970, avec l'exploration des potentialités du médium par les auteurs underground américains et par certains français et néerlandais. À ce laboratoire se substitue une standardisation de la production et la disparition de plusieurs titres innovants. Il termine son tour d'horizon par les espoirs suscités par la sortie et le succès de Maus contre la malédiction qui relègue encore la bande dessinée au rang d'art mineur sans potentialités.

Dans ce premier chapitre, comme dans les deux suivants, Menu propose une anthologie des auteurs les plus marquants du médium: il s'agit d'un premier travail de panthéonisation des auteurs considérés comme les précurseurs (Brétécher, Chaland, Crumb, Got, Gotlib, George Herriman, Mandryka, Moebius) du chemin qu'empruntera L'Association mais également des «bons » classiques franco-belges (Franquin, Macherot, Tilleux) voire d'auteurs que L'Association republiera (Baudoin, Forest, Gébé, Goossens, Masse, Tardi, Willem). Outre cette mise en avant des maîtres, Menu promeut le travail des fondateurs ou futurs auteurs de L'Association. Dupuy et Berbérian, Mattt Konture, Mokeït ou encore Lewis Trondheim ont le droit aux honneurs de son cours.

Le chapitre consacré à l'«approche ontologique » offre aux étudiants un éloge de la création libérée en bande dessinée. Là encore, l'idée d'une bande dessinée comme écriture est avancée. ${ }^{28}$ Pour Menu, le dessin n'a pas nécessairement à être très beau car il a avant tout valeur de signe. Comme en Egypte antique, écrire et dessiner sont des actions équivalentes : il s'agit d'un grand tout. Si Menu passe en revue la "grammaire » de la 
bande dessinée, il prend soin de distinguer entre les codes et les contraintes : le choix entre histoires courtes ou longues dans la narration s'explique avant tout par les impératifs économiques et technologiques des éditeurs. ${ }^{29} \mathrm{Ces}$ normes visent à faciliter la production et il serait possible d'interpréter l'histoire de la bande dessinée comme celle d'un long combat visant à s'affranchir des carcans éditoriaux. Pour Menu, il suffirait en effet de presque rien pour créer une narration en bande dessinée. Dans ce domaine, la technique a peu d'importance. ${ }^{30}$ Menu livre ainsi davantage des "trucs» et des considérations pragmatiques sur la technique qu'il ne cherche à imposer une façon de faire. Les extraits de planches parlent d'eux-mêmes. Dans le même ordre d'idées, la "bibliographie critique» est très succincte mais fait la part belle aux réflexions contemporaines engagées sur le médium (Lacassin, Fresnault-Deruelle, Rey, Lecigne, Groensteen, STP, Controverse et Dorénavant, mais également Les Cahiers de la bande dessinée ainsi que des monographies sur des auteurs en particulier). Le style assez relâché employé par Menu incite à découvrir par soi-même les potentialités du langage à partir d'un important recours à la richesse préexistante du médium.

Qu'il s'agisse du mémoire de maitrise (1988) ou du Cours de bande dessinée (1990), respectivement réédités en 2003 et 2007 comme cadeaux pour les adhérents, puis vendus dans L'Arrière-Boutique de L'Association, on ne peut qu'être frappé par la cohérence de la ligne adaptée par Menu. Le discours ne varie guère, il s'est matérialisé en partie dans les actes de L'Association et il donnerait presque le sentiment que ces deux livres auraient pu être réécrits après coup.

\section{La « voix » de L'Association}

\section{Faire ses preuves tout en entretenant la polémique (1990-2005)}

Du début des années 1990 jusqu'au milieu de la décennie suivante, le discours critique sur la bande dessinée de Menu se fait plus rare ou du moins plus discret. Il laisse la première place au "faire ". ${ }^{31}$ Les fondateurs de L'Association l'interprètent d'eux-mêmes comme une forme d'éclipse de l'aspect polémique de la maison d'édition..$^{32}$ Les six auteurs aux commandes de L'Association semblent alors tous s'accorder sur leur volonté d'aller vers une «bande dessinée créative », un « espace de liberté » où « expérimenter » et «tenter des trucs ${ }^{33}$ Son discours, bien que mis en sourdine, persiste néanmoins dans le paratexte produit par L'Association.

Les écrits de Menu dans ce contexte ne sont pas signés sauf lors de rares mises au point, notamment à la suite d'un désaccord avec Lewis Trondheim. Ce choix de Menu est influencé par la référence aux Situationnistes et s'explique également par l'idée d'un engagement collectif implicite autour de ses écrits qui donnent une voix à L'Association. Des années plus tard, des auteurs et anciens salariés de L'Association reconnaissent le rôle joué par Jean-Christophe Menu dans la mise en place d'une identité distinctive de l'éditeur. Mokeït parle à son sujet de " force de direction ». ${ }^{34}$

Ses écrits sont toutefois restreints à un public limité : le cercle étroit des adhérents et celui un peu plus large des lecteurs. L'éditorial constitue la forme reine du discours polémique de Menu, ce dernier réaffirmant les principes de la maison d'édition au gré de l'actualité de la structure et du monde de la bande dessinée. L'Association produit un paratexte, un discours d'accompagnement des œuvres, alors considérable pour un éditeur de bande dessinée: des catalogues de plus en plus conséquents chaque année et 
extrêmement soignés graphiquement, la feuille d'information des adhérents, le Rab' de Lapin, puis une forme élargie aux lecteurs et libraires, Les Nouvelles de l'Hydre, et bien sûr la revue Lapin. Ces différents supports paraissent plusieurs fois par an et à des fréquences plus ou moins fixes. Ils ont tous en commun de comporter un éditorial écrit par Menu. Dans celui-ci, Menu relie le plus souvent son discours sur la bande dessinée aux œuvres qu'il publie et construit progressivement la légitimité de L'Association au sein du champ de la bande dessinée. ${ }^{35}$

Son œuvre d'auteur de bande dessinée, notamment autobiographique, aborde également le sujet de la bande dessinée comme l'a bien analysé Erwin Dejasse dans sa comparaison du Livret de phamille avec deux autres autobiographies emblématiques de l'époque ( Approximativement de Lewis Trondheim et Journal d'un album de Dupuy et Berberian): "c'est d'abord et avant tout la bande dessinée qui constitue le sujet central. [...] La distinction - que l'on pouvait faire chez les générations qui les ont précédés - entre la création et les interventions externes n'a ici plus lieu d'être: œuvre et discours sur l'œuvre se confondent. ${ }^{36}$ Plus largement, les bandes dessinées que Menu édite alors à L'Association font preuve d'une grande conscience d'elles-mêmes, qu'il s'agisse du Journal d'un album ou de certaines œuvres de Baudoin qui interrogent l'image. Par ailleurs, les différentes livraisons de l'OuBaPo (OuPus) offrent une réflexion sur les particularités narratives de la bande dessinée et constituent un discours sur les potentialités de la bande dessinée.

Ses prises de position, ses choix éditoriaux et son œuvre d'auteur forment alors un tout indissociable. Menu fait à la fois œuvre d'auteur, de critique et d'éditeur. La synthèse de ces trois positions permet de rendre compte de la teneur de certaines œuvres publiées, assez classiques, et qui ne témoignent pas toutes de la radicalité entretenue et souhaitée par le discours de Menu, qui en tant qu'éditeur, adopte souvent une position moyenne qui peut apparaître paradoxale quand on la met en regard avec ses écrits d'alors. ${ }^{37}$

Contrairement à l'image de radicalité qui a pu lui être associée dans la seconde moitié des années 2000, Menu éditeur aura proposé une synthèse collective - reflétée par le catalogue de L'Association - de styles et de désirs différents, néanmoins unis autour de la volonté d'« ouvrir les limites» de la bande dessinée. Cette dynamique collective portée par le discours d'un seul homme se reconfiguerera cependant à partir des années 2000.

\section{L'Association dernière période (2005-2010) : polémique et théorie, individuel et collectif}

Lors de ce que l'on peut considérer comme L'Association dernière période (2005-2010) soit entre le départ d'une partie des fondateurs et celui de Menu - on assiste à un retour du discours critique sur la bande dessinée de la part de Jean-Christophe Menu. Par moment, ce discours peut sembler prendre le pas sur les œuvres publiées par l'éditeur. Cette radicalisation s'explique par la nouvelle place occupée par L'Association au sein du champ de la bande dessinée: l'éditeur a réussi à imposer ses auteurs (succès commerciaux et critiques de Lewis Trondheim, Joann Sfar, Emmanuel Guibert, David B. le plus souvent d'ailleurs chez d'autres éditeurs - et de Persépolis de Marjane Satrapi) et de nouveaux formats éditoriaux (noir et blanc, pagination libre, taille roman). Dès la fin des années 1990, L'Association fait des émules, aussi bien chez grands (« récupération » des formats éditoriaux et des auteurs du catalogue) que chez les petits éditeurs. ${ }^{38}$ Les innovations sont adoptées par d'autres, sans que ces derniers n'aient eu à porter les 
risques économiques. Ces éditeurs contribuent à brouiller le paysage - bien souvent avec le concours des auteurs de L'Association. Cette ouverture notamment chez des éditeurs autrefois classiques peut indéniablement être mise au crédit de L'Association. Elle ne va pas sans bousculer la singularité de l'éditeur en captant notamment une partie des œuvres qui auraient pu lui être destinées; en outre, elle engendre un certain conformisme qui fait redouter à Menu une répétition de l'académisme des années 1980. À cette concurrence pour les publics comme pour les auteurs (qui peuvent être " récupérés ») un autre facteur économique doit pris en compte ici : l'assise financière obtenue grâce au succès de Persépolis renforce l'indépendance de L'Association et lui permet d'approfondir ses expérimentations éditoriales et de radicaliser le discours qui les porte. En cela, L'Association ne fait que suivre une ligne revendiquée par exemple par un André Schiffrin pour qui l'éditeur se doit de financer des ouvrages plus difficiles avec ses succès commerciaux. ${ }^{39}$

Si la « récupération » est le détonateur d'un renouveau du discours critique, celui-ci n'avait jamais disparu et était présent bien avant les débuts de L'Association. Il est rendu plus visible à cette occasion et donne naissance à des développements qui n'auraient pas nécessairement pu avoir lieu dix ans plus tôt. L'essai Plates-bandes en donne le coup d'envoi et est prolongé par l'aventure polymorphe de L'Eprouvette, à la fois collection et revue théorique. D'une certaine façon, la thèse de doctorat de Jean-Christophe Menu vient clore ce mouvement alors même qu'il quitte L'Association. Sur cette période, le discours produit directement ou collectivement par Menu combine alors polémique et théorisation, même si le terme de systématisation semble mieux convenir.

\section{Plates-bandes : une critique du champ}

Comparant Plates-Bandes au Désoeuvré de Lewis Trondheim, qui paraît au même moment dans la même collection, Björn-Olav Dozo estime à juste titre que le premier reprend une grille de lecture bourdieusienne en l'appliquant au champ de la bande dessinée contemporaine. ${ }^{40}$ Menu comme Trondheim analysent les changements structurels qui viennent de traverser le champ de la bande dessinée, le premier de façon macrosociologique (au niveau des institutions, c'est-à-dire des éditeurs), le seconde de façon microsociologique (au niveau des auteurs). Dans les deux cas, pour Dozo, les auteurs s'interrogent sur la possibilité de l'existence d'une avant-garde en bande dessinée. Menu se place dans la continuité des manifestes littéraires du $\mathrm{xx}^{\mathrm{e}}$ siècle - le surréalisme en premier lieu - qui s'inscrivent eux-mêmes dans la tradition avant-gardiste. Celle-ci se caractérise par la revendication d'une originalité par les nouveaux entrants et cela depuis le mouvement romantique. ${ }^{41}$

Menu dresse un diagnostic peu reluisant de l'état du champ au début des années 2000, rappelant par bien des points le marasme des années 1980 qui avait suivi l'âge d'or des années 1960-1970. Les années 1980 avaient restreint l'espace des possibles, vu le triomphe du standard « 48CC » (l'album de 48 pages, en couleur et cartonné) alors que la bande dessinée s'isolait dans son ghetto de libraires spécialisés, de fans et de "spécialistes » sans grand sens critique. Pour Menu, une nouvelle vague de récupération serait désormais à l'œuvre.

Plates-Bandes permet de réaffirmer les principes à l'origine de L'Association et de la réunion de ce groupe d'auteurs. Selon le principal animateur de la structure, «la dimension de dissidence est essentielle dans la fondation de L'Association. » Ses fondateurs 
se retrouvent autour de deux motivations principales: «le désir d'utiliser la Bande Dessinée comme un moyen d'expression au sens absolu du terme » et la non-adéquation de ce désir avec le contexte professionnel de l'époque (la domination du « $48 \mathrm{CC}$ » qui ne permet plus l'expérimentation). ${ }^{42}$ Menu formalise donc a posteriori les principes présidant à la politique éditoriale de la structure, déjà présents dans le paratexte. Il les expose ici à un lectorat potentiellement plus large et souligne la contribution de L'Association au changement de perception de la bande dessinée par un nouveau public.

Outre cette exposition des principes, Menu se livre à l'exercice de la cartographie critique du champ ou pour reprendre l'idée de Dozo à un panorama de ce qui est lisible et de ce qui ne l'est pas. Pour ce faire, Menu adopte deux stratégies: ne pas nommer - et finalement nier la qualité - des auteurs et éditeurs des genres qu'il réprouve (il ne les désigne alors que sous la notion englobante de genre) et nommer individuellement les récupérateurs les plus proches, en n'hésitant pas à les énumérer. ${ }^{43}$ Pour Menu, «Il y a trois manières de récupérer : soit les Auteurs, soit les principes éditoriaux, soit, tant qu'à faire, tenter les deux à la fois. $»^{44}$ Le Seuil, précurseur mais extérieur au monde de la bande dessinée s'en tire bien, de même que la collection «Poisson Pilote» de Dargaud dirigée par Guy Vidal avec laquelle L'Association entretient "une cohabitation saine " puisqu'elle offre un renouvellement $d u$ « $48 \mathrm{CC} »$, contrairement à la collection "Ecritures» de chez Casterman, qui essaie de faire de L'Association (notamment en termes de format) à de meilleures conditions pour les libraires. La reprise de Futuropolis par Soleil est considérée comme le «scandale de l'an 2004 », ce qui se comprend aisément du fait du lien très fort qui unissait L'Association et Futuropolis, via « l'apprentissage » de Menu chez Robial et le transfert de certains auteurs de la seconde vers la première (Baudoin par excellence). ${ }^{45}$

40 Comment dépasser ce qui peut être vécu comme un grand bond en arrière? La bande dessinée semble alors sous le coup d'un rejet de son tournant intellectuel, qui risquerait de détourner certains du médium, alors même qu'une partie du capital symbolique accumulé par les Indépendants est récupéré par de gros éditeurs, même si leur discours critique reste inaudible. Pour Menu, la diffusion en librairie généraliste de la bande dessinée indépendante constitue l'une des solutions, avec la réaffirmation d'une certaine intransigeance, qui devrait se traduire concrètement par des œuvres toujours plus originales. Par le discours, Plates-Bandes se veut également une clarification d'une situation confuse du fait même de la publication d'auteurs de L'Association par les grands éditeurs. Comme l'a bien remarqué Tanguy Habrand, le thème de la « récupération » est ambivalent pour la structure puisque «Depuis sa fondation, L'Association oscille entre deux tendances à la fois complémentaires et contradictoires: "réveiller » et «être le réveil » de la bande dessinée. $»^{46}$ En conséquence, le succès obtenu par L'Association peut être lu comme une consécration de sa conception de la bande dessinée et de ses auteurs.

Plates-Bandes signe le renouveau du discours critique de L'Association et contribue à parachever la dislocation du comité éditorial de L'Association. Le départ de David B. en 2005 est en partie lié à la publication de cet ouvrage. En cherchant à cliver, à briser le « consensus mou » qui caractérise, à son avis, le champ de la bande dessinée, Menu ouvre implicitement un débat autour de la possibilité de vivre de la bande dessinée tout en restant fidèle à une conception exigeante de cette dernière. Pour la plupart des fondateurs, vivre de la bande dessinée était un objectif de départ que L'Association n'a jamais permis de réaliser pleinement en son sein. À leurs débuts, une part non négligeable des auteurs publie dans la presse catholique (Bayard, Fleurus), 
ponctuellement chez de gros éditeurs ou pratique l'illustration. Paradoxalement, si Menu n'est que très peu suivi dans cette voie du discours critique par la majorité des fondateurs - qui jugent tout à fait compatible bande dessinée d'auteur et gros éditeurs ${ }^{47}$ - il peut compter sur le renfort d'une nouvelle génération d'auteurs et de critiques dans le cadre de la collection et revue L'Eprouvette.

\section{L'Éprouvette : une entreprise collective de génération d'un discours}

L'Éprouvette naît en 2005 avec l'album Désœuvré de Lewis Trondheim, suivi du Plates-bandes de Jean-Christophe Menu. Si le projet d'une revue théorique était présent depuis déjà de nombreuses années à L'Association, ${ }^{48}$ la collection de livres (huit titres en 2010) précède la revue éponyme (trois numéros de 2006 à 2007). La création de ces deux supports éditoriaux découle de trois facteurs principaux. Avec le relatif éloignement des autres fondateurs, Jean-Christophe Menu finit de s'imposer définitivement comme l'éditeur du groupe et dispose d'une plus grande marge de manœuvre. Sa voix a toujours été prépondérante dans les choix éditoriaux mais il a désormais les moyens de proposer des ouvrages plus radicaux grâce à la manne financière apportée par le succès des livres de Marjane Satrapi. Enfin, Jean-Christophe Menu bénéficie du renouvellement générationnel des auteurs de bande dessinée. Une partie des nouveaux entrants et des auteurs historiques de L'Association répond cette fois à l'appel de la réflexion sur leur médium. Cette donnée tranche avec la fin des années 1980 et le début des années 1990 où les auteurs souhaitant s'exprimer sur le médium étaient encore rares. Ces nouvelles conditions rendent donc possible l'émergence d'une réflexion critique sur la bande dessinée qui émane de ses auteurs.

La collection de livres se distingue à la fois par ses bandes dessinées sur la bande dessinée et par ses essais qui adoptent un style littéraire pour traiter de la bande dessinée. La collection permet de faire éclore des livres traitant de la bande dessinée en bande dessinée. Il ne s'agit bien sûr pas d'une première comme l'œuvre de Scott McCloud (ou Menu) en témoigne. Certains albums autobiographiques n'ont pas pu éluder la question puisqu'ils sont dessinés par des praticiens. Dans les livres de L'Eprouvette, l'auteur reste le personnage principal mais son récit se concentre sur la bande dessinée. Les autobiographies précédentes abordaient avant tout le rapport de l'auteur à son médium et Désoeuvré de Trondheim s'inscrit d'ailleurs dans cette continuité. ${ }^{49}$ Les ouvrages de Baladi, Gerner et Mahler restent parfois réflexifs mais ils s'interrogent avant tout sur la représentation de la bande dessinée en tant que (sous)-culture au sein de leurs sociétés. $\mathrm{Si}$ Trondheim enquête sur le vieillissement de ses collègues et le caractère répétitif de la bande dessinée sur le mode de l'introspection, l'autrichien Mahler joue de l'absurdité de certaines réactions à l'encontre de la bande dessinée. Il décrit par exemple, avec une ironie amère, ses déboires avec sa perceptrice d'impôt qui n'arrive pas à envisager la bande dessinée comme de l'art. Dans une ribambelle de courts récits, qu'il poursuivra dans un second opus, Mahler se met scène face à l'incompréhension et au mépris déclenché par la bande dessinée en particulier dans les milieux culturels. ${ }^{50}$ Jochen Gerner s'inscrit dans une veine semblable avec son Contre la bande dessinée. Dans ce livre, il rassemble une collection de fragments vus et entendus au sujet de la bande dessinée. À partir de ces « brèves de comptoirs » appliquées à la bande dessinée - bien souvent issues d'ailleurs de sources réputées sérieuses - Gerner nous renseigne sur la perception de la bande dessinée et sur sa mauvaise réputation qui perdure. ${ }^{51}$ Dans Encore un effort, le suisse Alex Baladi se livre à une introspection sur la condition et le statut de l'auteur de bande 
dessinée..$^{52}$ Les bandes dessinées publiées dans cette collection ne se penchent pas tant sur la pratique en elle-même que sur son insertion sociale et sur une légitimité encore à acquérir.

Les essais sur la bande dessinée prenant la forme de textes sont moins nombreux mais ont la caractéristique d'être publiés par un éditeur de bande dessinée désireux de proposer également des textes. L'Association n'en est d'ailleurs par à son coup d'essai puisqu'elle a déjà édité la correspondance électronique d'Aleksandar Zograf, dessinateur serbe. Il ne s'agissait toutefois pas de bande dessinée mais d'un témoignage à chaud sur les bombardements occidentaux en Serbie. Outre le Plates-Bandes de Menu, la collection accueille une monographie de l'essayiste et réalisateur Pacôme Thiellement consacrée à l'œuvre de Mattt Konture. Christian Rosset, compositeur, producteur à France Culture et critique de bande dessinée propose quant à lui un recueil de textes dont une partie a déjà été publiée dans Les Cahiers de la bande dessinée, L'Année de la bande dessinée, $9^{e}$ Art ou L'Eprouvette. Dans cette "hantologie", dont le sous-titre est une référence à Derrida, Christian Rosset adopte un style très littéraire pour parler de bande dessinée. Rosset, qui collabore avec Menu sur un grand nombre d'entretiens dans la revue L'Eprouvette, publiera, dans cette même collection, une Corr\&spondance avec Menu à propos du rapport au corps en bande dessinée. ${ }^{53}$ Dans le domaine "textuel», la collection se distingue essentiellement par la qualité littéraire, presque poétique, des essais qu'elle publie.

Il en va de même pour la forme périodique de L'Éprouvette que Menu envisageait comme une "vraie revue littéraire ». Celle-ci constitue en partie l'aboutissement de ses désirs éditoriaux à L'Association :

C'est peut-être dans L'Éprouvette que j'ai le mieux exprimé tout ça: en faisant un éditorial Dada, en mettant les Situs un peu partout, en allant interviewer Carleman, Caradec, Roubaud. Il y avait quand même, associé à Pacôme Thiellement et à Christian Rosset, une vraie revue littéraire, qui s'intéressait à la bande dessinée mais qui était beaucoup plus ouverte que pouvait l'être le reste de L'Association. L'Éprouvette est née de cette limite que je commençais vraiment à sentir pesante au sein de L'Association : c'est-à-dire une routine de faire des livres de BD, et que nous ne soyons pas plus ouverts à autre chose. J'étais un peu seul à en avoir marre. Ça convenait très bien aux autres, qui étaient d'ailleurs de plus en plus absents. L'Éprouvette a contribué à tout faire exploser.

La politique éditoriale de L'Association a toujours été caractérisée par un grand soin apporté à l'ensemble de ses productions, du carton d'invitation au livre. ${ }^{54}$ Avec L'Eprouvette, la même exigence se retrouve et la revue dévoile ouvertement certaines influences extra-bande dessinée (des Situationnistes à l'Oulipo) avec par exemple des entretiens ou articles de Noël Arnaud, Jacques Roubaud ou une étude d'Antoine Sausverd sur les détournements de bande dessinée par les Situationnistes. La bande dessinée occupe bien sûr la place centrale dans ce dispositif, aussi bien dans les textes avec notamment une conscience historique des critiques passées, que dans les extraits de cases analysés par Erwin Dejasse comme une contribution à «l'histoire discriminante de la bande dessinée $» .^{55}$ Les trois numéros de la revue à la pagination croissante $(292$ p., 416 p. et $574 \mathrm{p}$.) proposent chacun un ou plusieurs dossiers thématiques composé(s) de textes et de planches. D'une très grande richesse en termes de contenu, la revue, que certains ont voulu résumer uniquement à la polémique, se saborde au troisième numéro (janvier 2006janvier 2007). bande dessinée. Le premier numéro s'intéresse ainsi au « système de la dédicace » comme 
symptôme du mercantilisme dominant le champ de la bande dessinée alors que le second prolonge la question avec le festival d'Angoulême. Dans les deux cas, ces thèmes ont fait l'objet d'un appel à contribution. Même si de nouveaux auteurs font leur apparition, comme Alex Baladi ou Morvandiau, de nombreux fidèles de L'Association apportent des planches, des textes voire les deux: Baudoin, Gerner, Guibert, Killoffer, Sfar, Sardon, Sattouf, Trondheim. Seuls David B., Mattt Konture et Stanislas manquent à l'appel parmi les fondateurs puisque Mokeït fait l'objet d'un long entretien dans le dernier numéro ( Le Septième Fondateur »). A travers les institutions de la bande dessinée, le public de celle-ci est visé comme en témoignent les nombreuses pages sur les "fans" pathologiques. Certains auteurs en prennent pour leur grade également comme «Les Fantasyens» de Riad Sattouf, caricature des dessinateurs d'heroic fantasy. Enfin, la correspondance publiée en fin de chaque numéro offre une critique féroce de la critique en bande dessinée, qui vise notamment l'ACBD (Association des critiques et journalistes de bande dessinée) et ses membres à l'occasion de la controverse opposant Lewis Trondheim, président du festival d'Angoulême, à Yves-Marie Labé. Dans ce dernier cas, Menu s'inspire, comme lors de certains échanges rapportés dans le Rab, de la correspondance de Guy Debord et Gérard Lebovici du temps des éditions Champs Libre.

L'Éprouvette continue le travail de Plates-bandes en chroniquant les dérives éditoriales en bande dessinée et cela dans chaque numéro via la rubrique «Plantes-bataille » où l'on retrouve des articles de Menu et d'autres critiques. De cette façon, L'Éprouvette continue à alimenter la controverse sur la « récupération » et plus généralement sur l'état du champ de la bande dessinée. Les pratiques des auteurs sont également dénoncées via des articles sur le plagiat (« La case la plus copiée » de Bernard Joubert et le dossier sur le « dispositif du plagiat »).

Deux autres caractéristiques du contenu de L'Éprouvette méritent enfin de retenir notre attention. Tout d'abord, la revue explore l'« érosion progressive des frontières » de la bande dessinée, c'est-à-dire les tentatives de rapprochement de cette dernière avec d'autres formes artistiques, ce qu'il est possible d'interpréter comme l'arrivée à une certaine maturité de la bande dessinée, moins soucieuse d'affirmer sa spécificité. Plus sûre de ses potentialités, et poussée par une logique de distinction qui l'oblige à toujours plus d'originalité, la bande dessinée expérimente en marge du champ. La photographie, l'art contemporain ou l'illustration sont présentés comme des possibilités non limitatives. Enfin, comme pour la réédition d'œuvres d'auteurs considérés comme des précurseurs de L'Association, L'Éprouvette offre un espace de patrimonialisation de la critique. Des textes de Bruno Lecigne (Controverse), Barthélémy Schwartz (Dorénavant), Etienne Robial ou encore Yves Di Manno sont réédités et des entretiens sont accordés le plus souvent à Menu, parfois en compagnie de Christian Rosset. Celui-ci signe quelques articles tout comme Pacôme Thiellement. Cet intérêt pour l'histoire de la critique vise à conforter la position avant-gardiste revendiquée par L'Association. Les contributions personnelles de Jean-Christophe Menu témoignent de cette réflexion sur l'avant-garde. Menu se fait l'historien et le comparse de ses précurseurs, Barthélémy Schwartz en premier lieu, lequel reprend goût à la bande dessinée grâce à L'Éprouvette. Menu établit des homologies entre avant-gardes quand il s'entretient sur le DIY (Do It Yourself) avec les Potagers Natures («Dialogue de DIY comparé»).

50 Concernant la revendication d'une avant-garde en bande dessinée, l'article de Menu sur «Avant-garde et ultracritique » est crucial. Il l'ouvre par deux citations, l'une de Tzara sur Dada et l'autre sur l'Internationale Situationniste à propos du Surréalisme. La 
généalogie est posée. Dans cet article, à la fois critique et historique, Menu s'interroge sur la mort des avant-gardes. Celle-ci aurait-elle un lien avec leurs accointances avec les idéologies politiques (fascisme pour le futurisme ou stalinisme pour le surréalisme) ? Le postmodernisme finirait de sceller leur sort. La chance de la bande dessinée reposerait paradoxalement dans son retard par rapport au reste de l'histoire de l'art. ${ }^{56}$ Elle peine à engendrer sa propre critique et ses praticiens rechignent à s'intéresser à son histoire. En bref, la bande dessinée pourrait être l'un des derniers arts disposant d'une avant-garde puisqu'elle n'a pas opéré sa modernisation. Dans cet article, Menu répond à l'affirmation du théoricien Harry Morgan qui estimait qu'une avant-garde n'avait pas de sens pour la bande dessinée. Pour Menu, l'expérimentation en bande dessinée est possible et consiste à repousser ses frontières. Nier la possibilité d'une avant-garde en bande dessinée revient à la cantonner au rang de sous-culture, à lui refuser une capacité à envisager des sujets non pas «adultes » mais «politiques ». L'avant-garde en bande dessinée se démarquerait par sa volonté « consciente » d'élargir le champ de la bande dessinée et par son recours à la théorie. Pour Menu, L'Association incarnerait la première avant-garde consciente du champ, puisqu'elle se serait positionnée comme telle face aux acteurs du microcosme, contrairement à ses prédécesseurs (Hara-Kiri, L'Écho des Savanes ou Métal Hurlant). Ces collectifs étaient finalement des avant-gardes incomplètes. Concernant l'absence d'un académisme en bande dessinée, Menu la réfute et estime que sa présence justifie d'autant plus la nécessité d'une avant-garde. Menu développe ensuite l'histoire de "l'Ultracritique » qu'il estime avoir continuée. Si l'Ultra-critique n'a pas été une avant-garde, « elle y a beaucoup ressemblé. ${ }^{57}$ Pour Menu, son travail se doit malheureusement d'être repris afin d'analyser les discours sur la bande dessinée et d'y déceler académisme et mercantilisme. Il convient de chasser la bande dessinée commerciale du champ de la bande dessinée au profit des artistes qui l'ont adopté comme langage et qui désirent repousser ses limites. L'Association mène finalement une lutte pour la définition de ce qu'est et devrait être la bande dessinée. Quitte à se répéter et à en éprouver de la lassitude, comme le remarque Menu dans le dernier numéro de L'Éprouvette («Platesbandes, extension finale («?») :

J'ai l'impression d'avoir écrit ce genre de lignes cent fois depuis vingt ans. Depuis le milieu des années 80 , les médiocres organisés qui confisquent à leur profit la Bande Dessinée ont changé de visage mais sont restés les mêmes. ${ }^{58}$

51 Pour Menu, L'Éprouvette aura cherché au cours de sa brève existence à être une " insulte " à la face du «microcosme», dont la logique économique semble malheureusement immuable.

Avec L'Éprouvette, Menu a révélé l'existence et a constitué un réseau d'auteurs et de critiques qui partagent une conception approchante et exigeante de la bande dessinée. « En mettant les auteurs au centre du discours critique (à la fois comme producteurs et comme objets), elle opère une révolution copernicienne: la bande dessinée quitte le monde des séries et des héros pour s'attarder sur les auteurs et leurs œuvres, comme en littérature ou en peinture. ${ }^{59}$ L'écho recueilli par L'Éprouvette en comparaison d'autres tentatives semblables (Critix, Comix Club) peut agacer les créateurs de ces dernières, ${ }^{60}$ mais cette réception différenciée s'explique vraisemblablement par le capital symbolique accumulé par L'Association en tant qu'éditeur prestigieux. Elle a le loisir de reconvertir ce capital dans le domaine de la critique, qu'elle n'avait de toute façon jamais vraiment abandonnée. 


\section{La bande dessinée et son double : un aboutissement et une ouverture}

53 Jean-Christophe Menu s'inscrit en thèse en 1990 et l'achève en octobre 2010 alors que L'Association fête son vingtième anniversaire. Les deux histoires sont intrinsèquement liées. Concomitant et inséparable de ses activités d'auteur et d'éditeur, ce doctorat aura à la fois été retardé et enrichi par celles-ci. Menu le conçoit en effet comme un retour sur une pratique et non comme un sésame pour le métier d'enseignant-chercheur.

Ces prémices expliquent la forme de la thèse. Dans celle-ci, Menu adopte la première personne du singulier tout au long de sa thèse comme le veut sa discipline (les arts plastiques). Groensteen «s'offusquer[a par exemple] de cet écrit par trop égocentré, égotique, autoréferentiel. » lors de la soutenance. ${ }^{61}$ Menu a toutefois cherché un temps, mais sans succès, à s'inscrire en esthétique afin de prendre de la distance avec son parcours d'auteur et d'éditeur. Ce lien entre théorie et pratique, cette obligation de «réflexion sur sa propre pratique», rend compte de la présence de planches, souvent déjà publiées ailleurs, et d'illustrations personnelles au sein du corps de la thèse de Menu.

Dans son introduction, Menu considère la bande dessinée comme sa langue maternelle : « je suis finalement en mesure de prétendre que ma première langue est bel et bien la Bande Dessinée, le Français étant, de fait, ma seconde langue. $»^{62}$ La bande dessinée est ici associée au monde de l'enfance à la fois via une lecture et une pratique assidue. Celle-ci revêt la forme d'une "écriture" libre ("une écriture directe, improvisée et automatique $»^{63}$. L'union ancienne entre Menu et le médium campe à la fois la conception de la bande dessinée de l'auteur et sa légitimité à en parler.

Le titre de sa thèse est un emprunt à Antonin Artaud, auteur du Théâtre et son double et l'une des grandes références de Menu. La thématique du double se veut être un «symbole de ce qui peut encore être réel et vrai dans l'Art». Menu dresse un parallèle entre la bande dessinée des années 1980 et le théâtre de boulevard dénoncé par Artaud dans l'Entre-deux-guerres. Cette forme théâtrale aurait amenuisé les potentialités réelles du théâtre, comme le « $48 \mathrm{CC}$ » celles de la bande dessinée, au profit du seul divertissement à but lucratif. L'idée de «Double» recouvre à la fois l'existant décevant de la bande dessinée et les potentialités liées à sa nature. Ainsi, la thèse n'explore pas seulement les perspectives futures mais dresse un bilan et envisage la possibilité de nouveaux dépassements des incarnations actuelles de la bande dessinée.

Du côté du bilan, Menu revient principalement sur le développement de l'autobiographie en bande dessinée et sur l'aventure OuBaPo. Il situe le tournant majeur de la première dans les années 1990, avec les auteurs de L'Association (Dupuy \& Berberian, Trondheim et lui-même) tout comme il reconnaît l'existence de précurseurs (de Crumb à Gotlib). Pour Menu, l'irruption du « moi » en bande dessinée est paradoxalement collective puisqu'elle émerge de façon simultanée dans une même structure. Quittant le mouvement collectif, Menu se penche ensuite sur sa propre pratique de l'autobiographie dans des développements assez personnels pour une thèse universitaire. Il met en garde contre l'avènement d'un nouveau genre en bande dessinée (l'autobiographie) comme le laissent craindre le succès des blogs d'auteurs. Menu range l'OuBaPo parmi les plus grandes avancées de la bande dessinée de ces deux dernières décennies : « Avec l'Autobiographie, le deuxième grand territoire vierge qui semblait riche de potentialités, au tournant des années 80 et 90, était la Potentialité même». Les précurseurs ou "plagiaires par anticipation » sont ici encore plus vénérables (Winsor McCay, George Herriman ou encore 
Gustave Verbeek). L'OuBaPo aura traduit les principes oulipiens en bande dessinée et avec la création d'objets-bande dessinée (BD-dés, SrOuBabble, DoMiPo) aura prouvé que «la Bande Dessinée est en mesure de produire des œuvres en trois dimensions sans que ne soit affecté son langage. $\aleph^{64}$. En conséquence, l'idée selon laquelle l'unité de la bande dessinée serait la case ressort renforcée de ces expérimentations. Dans une perspective approchante, Menu considère la bande dessinée au musée et les contraintes engendrées. L'«amusuéalité de la planche " prouverait ainsi en négatif les spécificités du médium alors que l'exposition "Toy Comix » attesterait des croisements fructueux qui peuvent être opérés entre bande dessinée et objets.

La deuxième partie de la sa thèse "Mythes et microcosme: au cœur de la bande dessinée » dresse un bilan du discours critique sur la bande dessinée. Menu revient sur son cours du CNED : «Ici aussi, mon but aura été de prouver qu'un cours de bande dessinée non seulement peut être réalisé en bande dessinée, mais prouve par là-même que ce langage est auto-suffisant pour parler de lui-même. " $^{65}$ Son activité d'éditeur n'est pas laissée de côté, au contraire. Il parle de L'Association comme d'un « double éditorial » où activité éditoriale équivaut à activité artistique. Il juge que L'Association représente davantage une "attitude" qu'un simple groupe. Celle-ci aurait été à l'avant-garde de 1990 à 1994 puis à nouveau à la fin des années 2000 avec l'épisode « Eprouvette ». En 2010, L'Association serait désormais une "institution $»^{66}$. Tout au long de son histoire, elle se serait efforcée d'opérer une "double rupture ", selon le terme de Bourdieu repris par Menu, à la fois avec la bande dessinée commerciale et avec la bande dessinée sociale. En cela, elle emprunterait la même voie que la littérature du xIX ${ }^{e}$ avec son ambition d'art pour l'art. Menu raconte l'histoire d'une structure destinée à remplir un vide qui s'est progressivement comblé. L'Association a commencé par publier l'impubliable mais une fois sa révolution réussie - l'élargissement de l'horizon éditorial - elle se retrouve confrontée à un problème économique qui découle de la perte de sa singularité.

Menu s'interroge aussi sur l'ambivalence et les contradictions de sa vision de la bande dessinée dans le chapitre "Traditions et ruptures». Il admet partager les mêmes références en bande dessinée que le « microcosme». Toutefois, contrairement à celui-ci, il cherche à les dépasser comme il le démontre avec des extraits d'exercices de réinterprétation graphiques de classiques comme Franquin, ou par l'hybridation et le détournement. Pour résumer, «De cette dialectique entre classicisme et rupture, seul est exclu le conformisme stérile et stéréotypé, préconçu commercialement par un microcosme et pour un marché, bref : l'académisme. $»^{67}$

Dans « Nouvelles perspectives internes ", Menu offre quelques réflexions à partir de son œuvre personnelle. Il forge le concept de «Monde clos » qui dépasse autobiographie et fiction et propose la « recherche personnelle d'un auteur pour élaborer la fiction qui lui ressemble le plus, sans souci de fournir au lecteur des repères rassurants et identifiables. $»^{68}$. Menu se fait également le défenseur de possibilités d'improvisation générées par le feuilleton, longtemps cantonné à sa fonction commerciale dans les magazines. Plus largement, Menu souligne l'importance des fragments dans son œuvre. En creux, ils nous informent sur sa conception de l'art: "La démarche fragmentaire correspond à mon intime conviction que le devoir minimum de l'artiste est de semer le doute, de décloisonner, de déverrouiller, et de faire entendre plusieurs voix. ${ }^{\prime 69}$

61 La troisième et dernière partie de sa thèse " Langage et frontières : au-delà de la bande dessinée » fait écho aux rubriques de la revue L'Éprouvette concernées par l'explosion progressive puis transgressive des frontières. Menu invite à aller vers les marges et vers 
d'autres formes artistiques afin d'éviter la "calcification ». Cette capacité à se mélanger serait inscrite dans la nature du médium : "Déjà composite à la base, le langage de la Bande Dessinée se prête de façon très productive aux hybridations les plus diverses. ${ }^{70}$. Ainsi, de cette manière, Menu exhorte à réconcilier l'histoire de l'art avec une histoire de l'art parallèle fondée sur le signe et incarnée, entre autres, par la bande dessinée. Cette recherche explique l'intérêt de Menu pour le "Corpus hors-champ de la bande dessinée ", par exemple les gouaches de Charlotte Salomon. En bref, il convient de se dégager des productions étiquetées comme de la bande dessinée par le champ pour des prétextes économiques. La lutte pour la définition de la bande dessinée continue.

Dans le dernier chapitre de sa démonstration («Théories du simple au double »), Menu évoque pour finir son rapport à la théorie. Une bonne théorie devrait être productrice de concepts pour nourrir la pratique. Il s'appuie sur une discussion des travaux de théoriciens comme Groensteen ou Fresnault-Deruelle pour estimer qu'une bande dessinée existe à partir de deux cases minimum. Elle se caractérise surtout par « l'espace feuilleté du livre » qui conduit Menu à attacher beaucoup d'importance aux éditeurs qui essaient de rapprocher leurs ouvrages des exigences et désirs des auteurs. Alors qu'il s'interroge sur le « Double » annoncé de la bande dessinée, Menu écarte la bande dessinée numérique des candidats, qu'il considère comme une simple antichambre la publication imprimée. Pour Menu, « la Bande Dessinée (désormais concept applicable à toute expérience Signe + Séquence) est donc le Double aussi bien de l'Art que de la littérature. $»^{71}$ En conclusion, Menu considère sa thèse comme une "poétique de la Bande Dessinée » appelée de ses vœux par Fresnault-Deruelle dès 1972.

63 Avec cette thèse, qui précède de quelques mois son départ de L'Association, JeanChristophe Menu devient le premier historien de L'Association après en avoir été le principal animateur. Il livre à la fois une synthèse collective et personnelle des expérimentations intervenues dans la bande dessinée de ces deux dernières décennies. Plus qu'une théorisation, il offre une démarche réflexive, ce qui a pu lui être reproché par Emmanuel Souchier, lors de sa soutenance, à travers notamment l'usage de certains termes. ${ }^{72}$ Ainsi, même s'il alimente son propos d'un grand nombre de références, notamment d'auteurs rangés sous l'étiquette de la French Theory (François Cusset), ${ }^{73}$ Menu revient surtout sur la cohérence d'un parcours de recherche en bande dessinée, c'est-àdire en actes. Par le discours, il imprime a posteriori une linéarité à ses choix éditoriaux et artistiques et opère un va-et-vient entre des réflexions générales sur l'évolution de la bande dessinée et son œuvre personnelle. La théorisation n'est pas complètement absente puisque la thèse articule un diagnostic des spécificités du langage avec un bilan et une perspective à partir de sa définition de la bande dessinée. Cette dernière fut éprouvée par la pratique personnelle de l'auteur et s'apparente donc peut-être par moment à l'essai, voire au manifeste, ou au témoignage. ${ }^{74}$ La thèse de Menu constitue toutefois une première pour un praticien de premier plan. Elle est d'autant plus riche qu'elle intervient une fois sa carrière d'auteur et d'éditeur bien remplie, sans préjuger de la coupure que constituera 2011 dans son parcours.

64 En définitive, lors de cette dernière période de renouveau d'un discours critique sur la bande dessinée, Menu prend le chemin de la polémique contre le retour du « consensus mou» tout comme il systématise ses réflexions sur la forme bande dessinée, via l'exploration des potentialités, et revendique le titre d'avant-garde pour L'Association. Sa démarche est alors tout autant collective avec L'Éprouvette qu'individuelle puisque sa thèse marque un moment-clé dans son parcours. 


\section{Cliver pour distinguer : la singularité du discours critique de Menu?} Les Cahiers de Mederologie de Guillaume Bordry et Nicolas Donin prennent pour objet son personnage Meder. ${ }^{75} \mathrm{~A}$ mi-chemin entre le fanzine et la revue universitaire, dont ils respectent les conventions (notes de bas de page, bibliographie, citations, «call for paper ", résumés en diverses langues dont le latin), Les Cahiers de Mederologie, sous-titré "Revue d'études sur MEDER de JC Menu ", connaissent deux numéros. Les auteurs des différentes contributions adoptent une graphie "mederienne" autant que possible et proposent des rubriques récurrentes: une étude de cas (analyses de planches, du vocabulaire employé, de l'usage de la trame et une étude de réception de Meder en Afrique), un élargissement des horizons («MEDER et le Moyen Age » de Silvère Menegaldo) et une «bibliothèque mederologique » dont la seule recension sera Histoire de la merde (1978) de Dominique Laporte.

Confidentielle et anecdotique, cette revue qui sera ensuite proposée à la vente aux adhérents de L'Association, offre donc un exercice de style académique au service de la parodie et de l'analyse poussée à l'absurde, une intertextualité débridée comme l'écrira en 2004 Donin dans un " Post-scriptum contextuel » présent dans l'ouvrage Munographie. ${ }^{76}$ Toutefois, en prenant pour objet d'analyse un personnage "punk» et de surcroît handicapé mental comme Meder, les auteurs des Cahiers soulignent, même parodiquement, que la bande dessinée peut très bien être un objet d'études sérieuses en dépit de la conclusion de l'article de Guillaume Bordry «La parole en révolte: l'onomatopée chez MEDER » :

Mais la plus éloquente de ces onomatopées nous parait être celle qu'il emploie, prenant le micro pour faire un discours, lors de la distribution des prix, à Angougou : «BLA ». Dans la droite ligne de toute l'aventure de MEDER à Angoulême, Jean-Christophe Menu, par l'intermeder de son personnage, offre un témoignage de la vanité du discours sur la bande dessinée, du discours en bande dessinée, et sans doute aussi du discours en général, et renvoie le lecteur au silence de sa lecture. ${ }^{77}$

Dans une veine plus sérieuse, Thierry Groensteen lui consacre un volume de sa collection «Etoiles de l'Image» en 2004 aux éditions de l'AN $2 .{ }^{78}$ Groensteen signe lui-même l'introduction ("Quelques traits de Jean-Christophe Menu ») où il ne tarit pas d'éloges sur celui-ci. Il le qualifie de "personnalité la plus emblématique des années quatre-vingtdix $\gg .{ }^{79}$ Le livre met plus l'accent sur l'œuvre d'auteur de Menu, mais Groensteen rattache l'ensemble de ses textes théoriques à son "œuvre militante ». A ce titre, il qualifie son mémoire de maîtrise de "l'un des plus fascinants témoignages de dessinateur dont nous disposons depuis les écrits de Töpffer ». Le livre comprend également des contributions de Guillaume Bordry, Nicolas Donin, Fabrice Neaud et Pacôme Thiellement sans compter les interventions de Menu lui-même, de la maquette à la publication d'inédits et à l'établissement d'une "Autobibliographie ». Dans les articles et mémoires au sujet de la bande dessinée datant des décennies 2000 et 2010, Menu est fréquemment cité notamment pour ses propos sur le « $48 \mathrm{CC}$ », expression passée dans le discours courant. À titre d'exemple, un outil aussi imparfait que Google Scholar donne déjà 16 citations pour son essai Plates-bandes, sachant que ce chiffre est largement sous-estimé. ${ }^{80}$ 
68 L'Association où la réception de son discours a pu être mitigée. Son discours a indéniablement réuni et été accepté tacitement par les autres fondateurs, les auteurs et une partie des salariés de L'Association au début de l'histoire de la structure. ${ }^{81} \mathrm{Au}$ fil des années, il a progressivement cessé de recouper totalement les convictions de l'ensemble des membres de L'Association. La première génération des auteurs de L'Association n'était pas très friande de théorisation. Lorsqu'ils sont interrogés, ces auteurs estiment que leurs œuvres constituent en elles-mêmes des manifestes sur leur conception de la bande dessinée. Ce n'est qu'avec une partie d'entre eux que Menu pourra mener à bien les projets autour de la collection et de la revue L'Eprouvette. Une nouvelle strate d'auteurs et de critiques, plus en phase avec ses convictions, le rejoindra à cette occasion. Mais chez la majorité des fondateurs, de l'acceptation assez consensuelle, puis tacite voire passive, on aboutira à un rejet de certains éléments de son discours, jugés trop extrêmes au milieu des années 2000. Dans cette optique, Pascal Ory juge ainsi qu'avec «le recul, on peut se demander si la principale réussite du groupe Menu ne tient pas, plutôt qu'aux valeurs collectives qu'affiche son leader, à la valeur individuelle que manifestent ses membres les plus doués. Vue sous cet angle, la contribution de L'Association à l'histoire du neuvième art s'apparente en effet à celle du surréalisme, dont les plus fortes personnalités, d'Aragon à Prévert en passant par Queneau, définitivement formées par cette expérience initiale, s'épanouirent en s'en éloignant. $»^{82}$

En dépit de son relatif isolement au sein des fondateurs et dans une certaine mesure des auteurs, le discours de Jean-Christophe Menu a recueilli un véritable écho dans les travaux sur la bande dessinée. Comment expliquer cette réception?

Au terme de notre démonstration, des éléments à la fois internes et externes au discours nous semblent apporter quelques clés de compréhension. Tout d'abord, le discours tenu par Menu est constant et cohérent tout au long de sa carrière. A ce sujet, Thierry Groensteen juge que Menu a donné à L'Association une « voix ». Ce faisant, il a singularisé L'Association en lui offrant un style « inimitable et reconnaissable entre tous » non dénué d'humour. Pour le compte de la maison d'édition, Menu a développé un point de vue de l'ordre du manifeste sur la bande dessinée en complément de ses pratiques éditoriales. Il a incarné par moments le rôle de "porte étendard de l'édition indépendante » avec ses prises de positions sur la grande édition. Pour Groensteen, «En ce sens-là, L'Association reste unique [...] indépendamment des livres qu'elle a fait et des succès qu'elle a eus, des auteurs qu'elle a révélés. $»^{83}$

71 Le radicalisme de la première période (début des années 1990) retrouve une nouvelle vie durant la dernière (mitan des années 2000). Ce discours volontairement provocateur reconnaît un certain nombre de contradictions, qu'il n'hésite pas à mentionner dans un certain nombre d'autocritiques, par exemple sur les choix de Menu d'être publié chez tel ou tel autre éditeur (participation à un Donjon chez Delcourt ou au magazine Spirou). Cette cohérence sur la durée explique le côté parfois très répétitif du discours de Menu : il n'a de cesse de dénoncer les mêmes travers du "microcosme». Les cibles changent seulement de nom d'une époque à l'autre (de Glénat à Soleil). Ces répétitions ont sûrement joué un rôle non négligeable dans le succès de son discours qui se voit alors repris quitte à être violemment critiqué..$^{84}$ En outre, le discours de Menu se démarque par la grande culture de son auteur, nourrie à la fois de ses lectures personnelles et de l'ouverture offerte par son parcours universitaire. Menu prend le soin de rattacher, voire de raccrocher, l'histoire de la bande dessinée à celle de l'art en général. Le Surréalisme et 
les Situationnistes sont les principales références mobilisées et elles cohabitent avec les précurseurs issus du champ de la bande dessinée, qu'ils soient auteurs ou critiques. Il a su jouer sur différents registres : il a prouvé le sérieux de son engagement pour la bande dessinée tout comme il a toujours veillé à insuffler une touche d'humour et d'autocritique à ses prises de position. Revenant sur la complexité de son parcours, Menu déclare :

il mélange des prises de position très claires et sans ambages. Les manifestes étaient vraiment sérieux, mais à côté de ça, j'ai toujours ressenti le besoin de mettre la petite connerie qui fait que l'on ne puisse pas prendre le manifeste trop au sérieux. C'est peut-être l'influence pataphysique, je ne sais pas, mais j'ai toujours mélangé et eu besoin de dialectique. Je déteste l'univocité.

Il faut ajouter à ce portrait l'évolution du positionnement de Menu sur le champ de la bande dessinée, d'auteur/éditeur marginal vers celui d'auteur/éditeur reconnu. La redécouverte de la polémique des années 1990 et le succès quasi-immédiat de celles des années 2000 doivent énormément à la position éditoriale solide qu'occupe alors Menu, principal animateur d'une maison d'édition qui a "découvert» les auteurs les plus talentueux des deux décennies qui viennent alors de s'écouler, et qui est associé au succès de Persépolis. Menu est alors en mesure de conserver et de faire fructifier le capital symbolique acquis en engageant L'Association dans une nouvelle phase de radicalisation. Celle-ci se traduit aussi bien dans les œuvres que dans le discours qui ne vise pas l'obtention d'un consensus. Enfin, à la fois auteur, éditeur et critique de bande dessinée, Jean-Christophe Menu est présent dans ces trois sphères, ce qui lui confère une maîtrise des différentes problématiques: il opère une synthèse d'une partie des discours précédemment portés par des acteurs différents. Sa position porte en fait plus sur la politique du champ de la bande dessinée, ses rapports de force et ses luttes définitionnelles, que sur l'esthétique du médium. Et dans le cas d'un champ encore instable comme celui de la bande dessinée, cela revient à parler d'économie et de standardisation de la production. En effet, à lire les prises de position de Menu, le champ semble encore sous la coupe de la logique économique, aux conséquences éditoriales et esthétiques néfastes, et n'a pas encore complètement réussi à imposer son autonomisation.

Dans ses prises de position, Menu se définit à la fois comme auteur et comme éditeur. Comme auteur d'abord, notamment lors de ses premiers écrits, où il déplore l'inexistence de structures éditoriales, mais aussi d'une critique en adéquation avec les désirs des auteurs de sa génération dont il se fait le porte-parole. Puis comme éditeur, une fois le succès venu, avec le thème de la "récupération ", et le reproche aux nouveaux arrivants ou à des éditeurs bien installés dans le champ de reprendre les principes de L'Association, son capital symbolique, pour les transformer en des ersatz lucratifs tout en reconnaissant que la confusion a été en partie occasionnée par une partie des auteurs de L'Association, dont lui-même dans une moindre mesure. Dans l'esprit de Menu, cette « récupération » menace de remettre en cause la survie économique de L'Association puisque ses auteurs travaillent avec des grands éditeurs, et puisque les nouveaux auteurs qui désirent marcher sur ses brisées ne sont plus cantonnés éditorialement à cette seule maison d'édition. Elle brouille également les repères, les hiérarchisations et les frontières que Menu a constamment tenté d'installer dans son discours. Cette « récupération » risque, et c'est finalement le plus grave aux yeux de Menu, d'engendrer une nouvelle phase de standardisation de la production (un nouvel académisme), cristallisée par la notion de « roman graphique ». Son œuvre d'éditeur solitaire, de 2005 à 2010, se sera efforcée de repousser les limites de la bande dessinée existante et de promouvoir toujours plus 
d'originalité, quitte à finalement abandonner ce qui constitue la bande dessinée aux yeux de certains acteurs du champ.

Le discours de Menu éditeur finit toutefois par rentrer en collision avec celui qu'il a pu tenir comme auteur : le champ a en partie évolué dans la direction souhaitée. Il permet désormais à des auteurs qui ne trouvaient pas de débouché pour leurs créations personnelles de s'épanouir chez de grands éditeurs qui leur accordent à la fois une liberté créative, notamment via des postes de directeurs de collection, et les moyens économiques de vivre de leur métier. Menu est en mesure de tenir un discours assez intransigeant puisqu'il parle avant tout comme éditeur, dans le sens où ses principaux revenus sont tirés de l'édition et non de son activité d'auteur. Pour le reste des auteurs, c'est l'inverse qui est vraie. Ils finissent d'ailleurs par se dégager pour la plupart de leurs activités éditoriales liées à L'Association. Ces auteurs, même s'ils bénéficient d'une certaine latitude dans leurs productions et occupent pour certains la position de directeurs de collection, deviennent matériellement dépendants des grands éditeurs.

La question de la possibilité de l'existence d'une avant-garde en bande dessinée devrait être reformulée afin de prendre en compte la dimension économique qu'évoquait par exemple Schwartz dans ses écrits : est-il possible de vivre de son art tout en ne concédant rien aux contraintes éditoriales ? Cette position d'auteur rémunéré par la bande dessinée est-elle compatible avec des exigences artistiques? Tout semble être une question d'où l'on place le curseur dans ce débat. Une partie des auteurs de L'Association estime tout à fait possible de concilier exigences éditoriales et publication chez de gros éditeurs. Un auteur comme Lewis Trondheim n'a jamais caché son désir de vivre de la bande dessinée tout comme finalement la majorité de ses contemporains à L'Association. Son statut d'auteur à succès et de directeur de collection chez Delcourt ne semble pas l'avoir restreint dans sa liberté artistique. Il en va de même pour David B. La position de Menu apparait plus stricte : il ne refuse pas aux auteurs de L'Association de publier chez de gros éditeurs, comme dans le cadre de la collection «Poisson Pilote» de Dargaud, mais leur reproche d'alimenter une forme de confusion. La publication de leurs livres chez des éditeurs multiples aux positionnements parfois antagonistes empêcherait le lecteur de s'y retrouver et aurait également des conséquences néfastes sur la présence en librairie des éditeurs alternatifs. Ces derniers ne sont pas en mesure d'offrir aux libraires les mêmes conditions que les gros éditeurs, même avec l'abandon de la vente ferme des albums.

La rareté des prises de parole publique sur l'état du champ de la bande dessinée par une partie des auteurs de L'Association a sûrement pour principale raison le désintérêt. ${ }^{85} \mathrm{La}$ position d'éditeur, avec un statut de salarié, ${ }^{86}$ permet paradoxalement plus de liberté dans la prise de parole afin de dénoncer les travers du champ de la bande dessinée. Ces attitudes relèvent également de conceptions différentes du champ : pour certains, il a assez évolué pour que l'on puisse investir le pôle de production de masse alors que pour d'autres, il convient de continuer à animer l'avant-garde. L'analogie avec la Révolution française peut être ici éclairante : les révolutionnaires qui estimaient que la Révolution avait atteint son but se transformaient en contre-révolutionnaire aux yeux de ceux qui jugeaient au contraire celle-ci encore incomplète. ${ }^{87} \mathrm{Il}$ semble en aller de même avec l'état du champ de la bande dessinée où chacun peut potentiellement se transformer en "contre-révolutionnaire " pour un autre en fonction du niveau perçu et toléré de compromis, voire de compromission. À cette incompatibilité supposée entre intégrité d'auteur et publication chez de gros éditeurs, il convient d'ajouter une autre fracture entre les statuts de juge et de partie. Menu, critique, pouvait-il cumuler cette casquette 
avec celles d'éditeur et d'auteur? Lorsque ses affirmations sont prises au mot, il devient difficile pour certain de comprendre pourquoi celui-ci s'autorise à publier chez de gros éditeurs, même très ponctuellement. Un discours axé sur l'intégrité artistique est-il cohérent avec l'activité d'éditeur, qui même si elle a lieu dans le cadre d'une association, génère des revenus $?^{88} \mathrm{~N}$ 'est-ce pas faire une lecture trop réductrice d'un discours qui a toujours su ménager des espaces de dérision et d'auto-critique que de le lire uniquement au premier degré? Le propre d'un discours provocateur n'est-il pas de simplifier pour amener le lecteur à réagir et à établir, dans le cas présent, des distinctions entre des œuvres guidées par l'originalité, et des produits suivant des recettes?

Dans cette optique, le discours critique tenu par Menu nous invite à relire certains travaux consacrés au champ de la bande dessinée. ${ }^{89} \mathrm{Si}$ l'on retient une définition du champ comme un espace d'affrontement entre des conceptions antagonistes de la bande dessinée, c'est-à-dire des jeux de définition, l'émergence d'un discours explicitant les positions des différents agents en présence contribue à révéler la lutte en cours. Briser le " consensus mou » conduit Menu à nommer, voire insulter, ceux qu'il considère comme ses adversaires : les éditeurs et auteurs dont il rejette les productions. Lorsqu'il sépare clairement la "Bande Dessinée ", notamment par l'emploi de cette orthographe, de la « BD », Menu invite le lecteur à distinguer au sein de la production et in fine à permettre des comparaisons entre des œuvres relevant de pratiques artistiques différentes. Pierre Bourdieu avait analysé l'autonomisation du champ artistique au xix ${ }^{e}$ siècle avec les exemples de Baudelaire et Flaubert..$^{90}$ Le champ littéraire s'était progressivement polarisé entre un pôle de production restreinte et un pôle de grande production. Le premier reconnaissait d'abord le jugement des pairs alors que le second se soumettait à la loi du marché. Par son discours et son œuvre d'éditeur, Menu cherche à jouer un rôle analogue au sein du champ de la bande dessinée. Son ambition est de contribuer à la formation du champ en le scindant entre des productions perçues comme délivrées de la contrainte économique, et donc libres d'explorer les potentialités de la bande dessinée, et des œuvres standardisées et destinées à répondre au goût du public.

\section{BIBLIOGRAPHY}

\section{Sources}

Les Cahiers de la bande dessinée, 1984-1988.

L'Éprouvette, 2006-2007.

Glob Off.

Labo, 1990.

Lynx, $\mathrm{n}^{\circ}$ 6-7.

BALADI. Encore un effort. Paris : L'Association, 2009. 80 p. Coll. « Eprouvette ». ISBN 9782844143068 
DAYEZ, Hugues. La nouvelle bande dessinée. Bruxelles : Niffle, 2004. 205 p. ISBN 9782873930288.

GERNER, Jochen. Contre la bande dessinée. Paris : L'Association, 2008. 136 p. Coll. « Éprouvette ». ISBN 9782844142559.

MAHLER, Nicolas. L'art selon madame Goldbruger. Paris : L'Association, 2005. 104 p. Coll. «Éprouvette ». ISBN 9782844141811.

MAHLER, Nicolas. L' art sans madame Goldgruber. Paris : L'Association, 2008. 112 p. Coll. «Éprouvette ». ISBN 9782844142726.

MENU, Jean-Christophe. Mémoire de maîtrise. Journal d'une existence de Bandes Dessinées. Paris : L’Association, 2003. 132 p. Coll. « Arrière-Boutique ». ISBN 9782844143327.

MENU, Jean-Christophe. Plates-bandes. Paris : L'Association, 2005. 80 p. Coll. «Éprouvette ». ISBN 9782844141644 .

MENU, Jean-Christophe. Cours de bande dessinée 1990, Paris, L'Association, 2007. 96 p. Coll. « Arrière-boutique ». ISBN 9782844142498.

MENU, Jean-Christophe et ROSSET, Christian. Corr\&spondance. Paris : L'Association, 2009. 40 p. Coll. « Éprouvette ». ISBN 9782844143242

MENU, Jean-Christophe. La bande dessinée et son double. Langages et marges de la bande dessinée. Paris : L'Association, 2011. 540 p. ISBN 9782844144256.

MENU, Jean-Christophe. « Cahier bande dessinée contemporaine ». Multitudes [en ligne], volume 52, n 1, 2013 [consulté le 28 avril 2015]. Disponible sur le Web. URL : <http:// www.cairn.info.scd-rproxy.u-strasbg.fr/revue-multitudes-2013-1-page-201.htm> DOI : <http:// dx.doi.org.scd-rproxy.u-strasbg.fr/10.3917/mult.052.0201>

MERCIER, Jean-Pierre. Entretien avec L'Association. Paris : L'Association, 2001. 82 p. Coll. « Arrièreboutique ». ISBN 9782844143518.

TRONDHEIM, Lewis. Désœuvré. Paris : L'Association, 2005. 72 p. Coll. «Éprouvette ». ISBN 9782844141620 .

\section{Ouvrages critiques et articles}

BEATY, Bart. Unpopular Culture. Transforming the European Comic Book in the 1990s. Toronto: University of Toronto Press, 2007. 320 p. ISBN 9780802094124.

BOLTANSKI, Luc. «La constitution du champ de la bande dessinée ». Actes de la recherche en sciences sociales [en ligne], volume 1, $\mathrm{n}^{\circ} 1,1975$ [consulté le 28 avril 2015]. Disponible sur le portail Persée. URL <http://www.persee.fr/web/revues/home/prescript/article/ arss_0335-5322_1975_num_1_1_2448> DOI <10.3406/arss.1975.2448>

BORDRY, Guillaume et DONIN, Nicolas. Les Cahiers de Mederologie. Revue d'études sur MEDER de J-C Menu, 2001-2002.

BOURDIEU, Pierre. Les règles de l'art. Genèse et structure du champ littéraire. Paris : Le Seuil, 1992. 480 p. ISBN 2020181592.

CARACO, Benjamin. « La communication éditoriale : un outil de légitimation. Le cas de L'Association », Comicalités [En ligne], Théorisations et médiations graphiques, 2013 [consulté le 28 avril 2015]. Disponible sur le Web. URL : <http://comicalites.revues.org/1707> ; DOI : <10.4000/ comicalites.1707> 
DEJASSE, Erwin. «Les discours sur la bande dessinée par ses créateurs », Publif@rum [En ligne], n - 14, 2011 [consulté le 28 avril 2015]. Disponible sur le Web. URL : <http://publifarum.farum.it/ ezine_articles.php?art_id=194>

DEJASSE, Erwin. « Sacralisation et légitimation. Les hors-textes de la revue L'Éprouvette :

fragments d'une Histoire discriminante. ». Comicalités [En ligne], La bande dessinée : un « art sans mémoire ? », 2012 [consulté le 28 avril 2015]. Disponible sur le Web. URL : <http:// comicalites.revues.org/1093> DOI <10.4000/comicalites.1093>

DONY, Christophe, HABRAND, Tanguy et MEESTERS, Gert (dir.). La bande dessinée en dissidence : alternative, indépendance, auto-édition / Comics in Dissent: Alternative, Independence, Self-Publishing. Liège : Presses Universitaires de Liège, 2014. 218 p. ISBN 9782875620385.

DOZO, Björn-Olav. « La Bande dessinée francophone contemporaine à la lumière de sa propre critique. Quand une avant-garde esthétique s'interroge sur sa pérennité », Belphégor [En ligne], volume 6, n 2, 2007 [consulté le 28 avril 2015]. Disponible sur le Web. URL : < http:// dalspace.library.dal.ca/handle/10222/47737 >

GROUPE ACME. L'Association : une utopie éditoriale et esthétique. Bruxelles : Les Impressions nouvelles, 2011. 221 p. ISBN 2874491238.

HABRAND, Tanguy. « La "récupération" dans la bande dessinée contemporaine. Par-delà récupérateurs et récupérés ». Textyles : Revue des Lettres Belges de Langue Française [en ligne], volume 36-37, 2010 [consulté le 28 avril 2015]. Disponible sur le Web. URL : <http:// textyles.revues.org/1417>

MENU, Jean-Christophe. Munographie. Angoulême : Editions de l'AN 2, 2004. 128 p. ISBN 9782848560168.

MORGAN, Harry. « Le discours sur la bande dessinée. Bilan historique. 1830-1970 », The Adamantine [En ligne], 2007 [consulté le 28 avril 2015]. Disponible sur le Web. URL : <http:// theadamantine.free.fr/univete.html>

ORY, Pascal et MARTIN, Laurent (dir.). L'art de la bande dessinée. Paris : Citadelles \& Mazenod, 2012. 586 p. ISBN 9782850885310.

POOT, Christophe. «(syn)thèse ». du9, l'autre bande dessinée [En ligne], janvier 2011 [consulté le 28 avril 2015]. Disponible sur le Web. URL : <http://www.du9.org/humeur/syn-these/>

SAPIRO, Gisèle. La sociologie de la littérature. Paris : La Découverte, 2014. 128 p. Coll. « Repères ». ISBN 9782707165749.

SCHIFFRIN, André. L'édition sans éditeurs. Paris : La Fabrique, 1999. 94 p. ISBN 9782913372023.

\section{NOTES}

1. Christophe Dony, Tanguy Habrand et Gert Meesters (dir.), La bande dessinée en dissidence: alternative, indépendance, auto-édition / Comics in Dissent: Alternative, Independence, Self-Publishing, Liège, Presses Universitaires de Liège, 2014, p. 29.

2. Hugues Dayez, La nouvelle bande dessinée, Bruxelles, Niffle, 2004.

3. Benjamin Caraco, «La communication éditoriale: un outil de légitimation. Le cas de L'Association ", Comicalités. Études de culture graphique, septembre 2013.

4. Jean-Christophe Menu, Plates-bandes, Paris, L'Association, 2005.

5. Jean-Christophe Menu, La bande dessinée et son double: langages et marges de la bande dessinée, Paris, L'Association, 2011. 
6. Ce travers n'est pas nouveau si l'on en croit Dejasse: «Dans les premières revues d'étude consacrées à la bande dessinée, la voix des auteurs se fait certes entendre mais, quasi exclusivement, par le biais d'interviews: ce sont des discours suscités quii sont d'un intérêt très variable selon ce que l'auteur a à dire ou souhaite exprimer. » Erwin Dejasse, « Les discours sur la bande dessinée par ses créateurs », http://publifarum.farum.it/, février 2011.

7. Harry Morgan, «Le discours sur la bande dessinée. Bilan historique. 1830-1970», The Adamantine, URL : http://theadamantine.free.fr/univete.html

8. Tanguy Habrand, "La "récupération" dans la bande dessinée contemporaine ", Textyles. Revue des lettres belges de langue française, juin 2013, p. 75-90.

9. Björn-Olav Dozo, «La Bande dessinée francophone contemporaine à la lumière de sa propre critique. Quand une avant-garde esthétique s'interroge sur sa pérennité », Belphégor, vol.6 / 2, 2007.

10. Erwin Dejasse, op. cit.

11. Entretien du 15 avril 2014, Paris.

12. Groupe Acme, L'Association: une utopie éditoriale et esthétique, Bruxelles, Les Impressions Nouvelles, 2011, p. 13.

13. Il fait référence à la collection « $X$ » de l'éditeur Futuropolis où Menu publie l'un de ses premiers albums en compagnie de certains des futurs fondateurs et auteurs de L'Association (Mokëit, Stanislas, Vanoli).

14. Collectif, Labo en bande dessinée, Paris, Futuropolis, 1990, p. 91-93.

15. Ibid., p. 93.

16. Auquel il faut ajouter le texte de Barthélémy Schwartz qui relève également du discours critique sur la bande dessinée dans ce même numéro de Labo.

17. Ibid., p. 91.

18. Jean-Christophe Menu, Mémoire de maîtrise. Journal d'une existence de Bandes Dessinées, Paris, L'Association, 2003.

19. La bibliographie du mémoire comporte des ouvrages d'Artaud, Breton et Jarry.

20. Ibid., p. 9.

21. Il fait de cette dernière l'« étalon-or de toute ma création ». (p. 49)

22. Ibid., p. 48.

23. Ibidem, p. 56.

24. Association pour l'Apologie du Neuvième Art Libre.

25. Jean-Christophe Menu, Cours de bande dessinée 1990, Paris, L’Association, 2007.

26. Will Eisner, Les clés de la bande dessinée, Paris, Delcourt, 2009.

27. Scott McCloud, L'art invisible, Paris, Delcourt, 2007.

28. Jean-Christophe Menu, op. cit., p. 39.

29. Ibidem, p. 54 .

30. Ibidem, p. 63.

31. J.-C. Menu, « Cahier bande dessinée contemporaine », Multitudes, vol. 52 / 1, juin 2013, p. 206.

32. Jean-Pierre Mercier, L'Association. Entretien avec Jean-Pierre Mercier, Paris, L'Association, 2000, p. 23-24.

33. Ibidem, p. 24.

34. Entretien avec Mokeït, 21 avril 2014.

35. Pour une analyse de ces supports, cf. Benjamin Caraco, op. cit.

36. Groupe Acme, op. cit., p. 82 et 91.

37. Entretien avec Jean-Christophe Menu, 15 avril 2014.

38. Tanguy Habrand, op. cit.

39. André Schiffrin, L'édition sans éditeurs, trad. Michel Luxembourg, Paris, La Fabrique éd., 1999.

40. Björn-Olav Dozo, op. cit.

41. Gisèle Sapiro, La sociologie de la littérature, Paris, La Découverte, p. 24. 
42. Ibidem, p. 25.

43. Björn-Olav Dozo, op. cit.

44. Jean-Christophe Menu, op. cit., p. 35.

45. « Futuropolis a été et demeure le principal modèle d'éthique et d'intransigeance pour tous les éditeurs dits Indépendants apparus depuis 1990, à commencer par L'Association, qui peut s'en estimer en filiation directe à bien des titres. ", Ibidem, p. 41.

46. Tanguy Habrand, op. cit., p. 84.

47. Bart Beaty, Unpopular culture: transforming the European comic book in the 1990s, Toronto (CA), University of Toronto Press, 2007, p. 152.

48. Dans sa contribution au premier numéro de l'Eprouvette, Joann Sfar mentionne un tel projet dès « 1994 ou 1995 » lors d'une assemblée générale de L'Association (L'Association, L'Eprouvette, $\mathrm{n}$ 1, p. 1, 27.).

49. Lewis Trondheim, Désoeuvré: essai, Paris, L'Association, 2005. Pour une analyse du livre, cf. Björn-Olav Dozo, op. cit.

50. Nicolas Mahler, L'art selon madame Goldbruger: insulte, trad. Waltraud Spohr, Paris, L'Association, 2005. Nicolas Mahler, L' art sans madame Goldgruber: saillies, Paris, L'Association, 2008.

51. Jochen Gerner, Contre la bande dessinée : choses lues et entendues, Paris, L'Association, 2007.

52. Baladi, Encore un effort: fidèle coutelas, Paris, L'Association, 2009.

53. Le livre est coédité par le festival rennais "Périscopages». Christian Rosset et JeanChristophe Menu, Corr\&spondance, Paris, L'Association, 2009.

54. Groupe Acme, op. cit., p. 11-31.

55. Erwin Dejasse, "Sacralisation et légitimation. Les hors-textes de la revue L'Éprouvette: fragments d'une Histoire discriminante », Comicalités. Études de culture graphique, septembre 2012. 56. Son éditorial à la Dada exprime cette thèse avec humour dans le premier numéro.

57. L'Association, op. cit., $\mathrm{n}^{\circ} 1, \mathrm{p} .185$.

58. Ibidem, $\mathrm{n}^{\circ} 3$, p. 446.

59. Groupe Acme, op. cit., p. 43.

60. Comme l'illustre les planches de Big Ben, éditeur du Comix Club, dans le second numéro de $L^{\prime}$ Eprouvette.L'Association, op.cit., $\mathrm{n}^{\circ} 2$, p. 261-263. Ces planches démontrent encore une fois l'autocritique tolérée par Menu au sein même de ses publications.

61. Christophe Poot, "(syn)thèse ", du9, l'autre bande dessinée.

62. Jean-Christophe Menu, op. cit., p. 21.

63. Ibidem, p. 23.

64. Ibidem, p. 135.

65. Ibidem, p. 178.

66. Ibidem, p. 187.

67. Ibidem, p. 278.

68. Ibidem, p. 289.

69. Ibidem, p. 335.

70. Ibidem, p. 366 .

71. Ibidem, p. 483.

72. «Emmanuel Souchier dissèque à son tour le modus operandi de Menu. S'il lui trouve les vertus littéraires et personnelles de la thèse d'un homme qui fait corps avec le sujet de son étude, il pointe douloureusement ce que ce modus operandi n'est pas : soit un travail qui pose non pas un Sujet, mais un Objet. Un objet à examiner sous toutes les coutures, sans affect, sans pré-requis, sans préjugés, comme une Chose étrangère à soi. » in Christophe Poot, op. cit.

73. La bibliographie de sa thèse comporte des titres d'auteurs comme Boltanski, Barthes, Bataille, Blanchot, Bourdieu, Genette, Lejeune et Lyotard.

74. Christophe Poot, op. cit. 
75. Il faut sûrement y voir une référence à la « médiologie », discipline contemporaine...

76. Collectif, Munographie, Angoulême, Editions de l'AN 2, p. 45.

77. Guillaume Bordry et Nicolas Donin, Les Cahiers de Mederologie. Revue d'études sur MEDER de JCMenu, 2000, p. 15 ( $\left.\mathrm{n}^{\circ} 1\right)$.

78. Collectif, op. cit.

79. Ibidem, p. 5.

80. Consulté le 24 octobre 2014.

81. Les premiers salariés sont engagés dès 1994.

82. Pascal Ory et Laurent Martin (dir.) L'art de la bande dessinée, Paris, Citadelles \& Mazenod, p. 352.

83. Entretien avec Thierry Groensteen, 13 juin 2014 à Paris.

84. On peut penser aussi aux controverses l'opposant aux rédacteurs du site « Actuabd».

85. À cela il conviendrait sûrement d'ajouter une forme d'auto-censure ou un manque de confiance dans sa légitimité à s'exprimer sur le médium.

86. Menu n'est toutefois pas le salarié de L'Association sur toute cette période, notamment au début puis peu avant son départ.

87. Jean-Clément Martin, Contre-révolution, révolution et nation en France: 1789-1799, Paris, Éd. du Seuil,1998.

88. Bruno Lecigne juge ainsi incompatible critique et édition.

89. Luc Boltanski, "La constitution du champ de la bande dessinée ", Actes de la recherche en sciences sociales, vol. 1 / 1, 1975, p. 37-59.

90. Pierre Bourdieu, Les règles de l'art: genèse et structure du champ littéraire, Paris, Ed. du Seuil, 1992.

\section{INDEX}

Mots-clés: bande dessinée, légitimité, roman graphique, nouvelle bande dessinée, L'Association

\section{AUTHOR}

\section{BENJAMIN CARACO}

Conservateur des bibliothèques et doctorant en histoire contemporaine à l'Université Paris-1, Benjamin Caraco travaille sur l'histoire de L'Association, maison d'édition de bande dessinée indépendante. Sur ce sujet, il a publié « La communication éditoriale : un outil de légitimation. Le cas de L'Association » dans la revue Comicalités en 2013. 